\title{
Robust Stability Analysis for Interval Cohen-Grossberg Neural Networks With Unknown Time-Varying Delays
}

\author{
Huaguang Zhang, Senior Member, IEEE, Zhanshan Wang, and Derong Liu, Fellow, IEEE
}

\begin{abstract}
In this paper, robust stability problems for interval Cohen-Grossberg neural networks with unknown time-varying delays are investigated. Using linear matrix inequality, $M$-matrix theory, and Halanay inequality techniques, new sufficient conditions independent of time-varying delays are derived to guarantee the uniqueness and the global robust stability of the equilibrium point of interval Cohen-Grossberg neural networks with time-varying delays. All these results have no restriction on the rate of change of the time-varying delays. Compared to some existing results, these new criteria are less conservative and are more convenient to check. Two numerical examples are used to show the effectiveness of the present results.
\end{abstract}

Index Terms-Cohen-Grossberg neural networks, Halanay inequality, interval neural networks, linear matrix inequality (LMI), $M$-matrix, robust stability, time-varying delays.

\section{INTRODUCTION}

$\mathbf{S}$ INCE Cohen and Grossberg proposed a class of neural networks in 1983 [12], this model (called Cohen-Grossberg neural networks) has received increasing attention due to its promising potential for applications in pattern formation and associative memories. This kind of Cohen-Grossberg neural networks include Hopfield neural networks [18], shunting neural networks, and other neural networks [14], [15] as special cases. Compared to Hopfield neural networks, the advantages of Cohen-Grossberg neural networks are as follows.

1) Cohen-Grossberg neural networks represent a large kind of biological neural systems, including a number of models from population biology, neurobiology, and evolutionary theory, especially the Hopfield neural

Manuscript received March 09, 2008; revised July 08, 2008; accepted July 16, 2008. Current version published November 05, 2008. This work was supported by the National Natural Science Foundation of China under Grants 60534010, 60572070, 60728307, 60774048, and 60774093, by the Program for Cheung Kong Scholars and Innovative Research Groups of China under Grant 60521003 , by the National High Technology Research and Development Program of China under Grant 2006AA04Z183, by the 111 Project of China Ministry of Education (B08015), by the Natural Science Foundation of Liaoning Province under Grant 20072025, and by the Postdoctoral Foundation of Northeastern University under Grant 20080314.

$\mathrm{H}$. Zhang and Z. Wang are with the School of Information Science and Engineering, Northeastern University, Shenyang, Liaoning 110004, China and also with the Key Laboratory of Integrated Automation of Process Industry (Northeastern University), China Ministry of Education, Shenyang, Liaoning 110004, China (e-mail: hgzhang@ieee.org; zhanshan_wang@163.com).

D. Liu is with the Department of Electrical and Computer Engineering, University of Illinois at Chicago, Chicago, IL 60607 USA (e-mail: dliu@ece.uic. edu).

Digital Object Identifier 10.1109/TNN.2008.2006337 networks as a special case. Cohen-Grossberg neural networks are more general than Hopfield neural networks in the aspects of structure and implementation.

2) The dynamics of Cohen-Grossberg neural networks can reflect the evolution of biological system and population model, in which the equilibrium point must be nonnegative, representing the case of species' competition and extinction. In some cases of amplification function, Cohen-Grossberg neural networks can have the same dynamics as that of Hopfield neural network. However, the dynamics of Hopfield neural network generally cannot reflect the evolution of biological system and population model, for example, Lotka-Volterra system.

Because Cohen-Grossberg neural networks have been successfully applied in classification, parallel computation, associative memories, and especially in solving optimization problems, research on Cohen-Grossberg neural network has recently attracted much attention and has become a hot topic in the neural network research community. Such applications rely on the qualitative stability properties of the network. Thus, the qualitative analysis of the network's dynamic behavior is a prerequisite step for the practical design and application of neural networks. Because of the finite speed of switching and transmission of signals, time delays are inevitably present in electronic implementation of neural networks, which can influence the stability of the entire network by creating oscillatory or unstable behaviors. Recently, some sufficient conditions for the global asymptotic and exponential stability of delayed Cohen-Grossberg neural networks have been established in [3], [9]-[11], [16], [19], [20], [27]-[29], [32], [35]-[37], [42], and [47]. However, in electronic implementation of neural networks, there also exist inevitably some uncertainties due to the existence of modeling errors and parameter fluctuations, which lead to complex dynamical behaviors. Thus, a good neural network design should have robustness against such uncertainties. In [26], the authors investigated the robust stability problem for a kind of interval delayed neural networks and have obtained a sufficient condition for robust stability of the unique equilibrium point. For the interval neural network models similar to that in [26], some robust stability results are derived in [2], [5], [8], [21], [25], and [34] based on $M$-matrix theory or some algebraic inequality approaches. Recently, some global robust exponential stability criteria were proposed in [11], [29], and [35] for interval Cohen-Grossberg neural networks with time-varying delays based on differential inequality techniques and $M$-matrix theory. Some linear 
matrix inequality (LMI)-based criteria are proposed in [32] for uncertain Cohen-Grossberg neural networks with constant delay. Although the suitability of the criteria based on differential inequality techniques is improved significantly, these criteria are generally difficult to check because there are many parameters to be tuned. Today, the LMI technique has been successfully employed in tackling many stability problems of neural networks. The derived stability criteria can efficiently be solved using the interior point algorithms, and are less conservative than some previous results [10], [17], [21]-[24], [33], [34], [39], [44]. However, for the case of time-varying delays, the LMI-based stability results usually assume that the value of derivative of time-varying delays is less than one [1], [3], [6], [8], [11], [13], [17], [23], [24], [38], [43], which seriously restricts the application of the corresponding results, especially in the case of unknown time-varying delays.

In this paper, we investigate the global robust stability of equilibrium point for interval Cohen-Grossberg neural networks with unknown time-varying delays based on the LMI technique under the assumption that the amplification function is continuous, positive and bounded. Moreover, we also present a global robust stability condition for the positive equilibrium point of interval Cohen-Grossberg neural networks with unknown time-varying delays under the assumption that the amplification function is continuous and nonnegative. All the obtained results are independent of time-varying delays, which also means that we do not restrict the value of the derivative of the time-varying delays.

This paper is organized as follows. Problem statement and preliminaries are given in Section II. Global robust exponential stability results independent of time-varying delays are presented in Section III. Some sufficient conditions for the existence and uniqueness, and the global robust stability of the concerned Cohen-Grossberg neural networks with positive equilibrium point are presented in Section IV. Two numerical examples are utilized to demonstrate the validity of the obtained results in Section V, and conclusions are given in Section VI.

\section{Problem Statement AND Preliminaries}

Consider the following interval Cohen-Grossberg neural networks with multiple time-varying delays:

$$
\begin{aligned}
\frac{d u_{i}(t)}{d t}=-d_{i}\left(u_{i}(t)\right) & {\left[a_{i}\left(u_{i}(t)\right)-\sum_{j=1}^{n} w_{0 i j} \bar{g}_{j}\left(u_{j}(t)\right)\right.} \\
& \left.-\sum_{j=1}^{n} w_{1 i j} \bar{f}_{j}\left(u_{j}\left(t-\tau_{i j}(t)\right)\right)+U_{i}\right]
\end{aligned}
$$

where $u_{i}(t)$ denotes the state of the $i$ th neuron at time $t$, $d_{i}\left(u_{i}(t)\right)$ denotes the amplification function, $a_{i}\left(u_{i}(t)\right)$ denotes the appropriately behaved function such that the solution of the model given in (1) remains bounded, $\bar{g}_{j}\left(u_{j}(t)\right)$ and $\bar{f}_{j}\left(u_{j}(t)\right)$ denote the activation function, $\tau_{i j}(t) \geq 0$ are bounded and unknown time-varying delays, $\rho=\max \left(\tau_{i j}(t)\right)$, $W_{0}=\left(w_{0 i j}\right)_{n \times n}$, and $W_{1}=\left(w_{1 i j}\right)_{n \times n}$ denote the connection weight and delayed connection weight matrices, respectively, $\underline{w}_{0 i j} \leq w_{0 i j} \leq \bar{w}_{0 i j}, \underline{w}_{1 i j} \leq w_{1 i j} \leq \bar{w}_{1 i j}$, and $U_{i}$ denotes the external constant input bias, $i, j=1, \ldots, n$. The initial condition is $u_{i}(\varsigma)=\phi_{i}(\varsigma)$, where $\varsigma \in[-\rho, 0]$, and $\phi_{i} \in C([-\rho, 0], \Re)$ denotes a continuous and bounded set from $[-\rho, 0]$ to $\Re$ with $\bar{\phi}=\sup _{-\rho \leq \varsigma \leq 0}\|\phi(\varsigma)\|, i=1, \ldots, n$.

Throughout this paper, let $\bar{B}_{k}=\left(b_{i j}^{k}\right)_{n \times n}$, whose $k$ th row is composed of the $k$ th row of matrix $W_{1}=\left(w_{1 i j}\right)_{n \times n}$ and other rows are all equal to zero, $\underline{w}_{1 k j} \leq b_{k j}^{k}=w_{1 k j} \leq \bar{w}_{1 k j}$. For a real square matrix $D, D<0(>0)$ denotes a symmetric negative(positive)-definite matrix, $D^{T}, D^{-1}, \lambda_{m}(D)$, and $\lambda_{M}(D)$ denote the transpose, the inverse, the smallest eigenvalue, and the largest eigenvalue of matrix $D$, respectively. Let $\|D\|$ denote the Euclidean norm defined by $\|D\|=\sqrt{\lambda_{M}\left(D^{T} D\right)}$. Let $U=\left(U_{1}, \ldots, U_{n}\right)^{T}, \underline{W}_{0}=\left(\underline{w}_{0 i j}\right)_{n \times n}, \bar{W}_{0}=\left(\bar{w}_{0 i j}\right)_{n \times n}$, $\underline{W}_{1}=\left(\underline{w}_{1 i j}\right)_{n \times n}, \bar{W}_{1}=\left(\bar{w}_{1 i j}\right)_{n \times n}, W^{+}=\left(\bar{W}_{0}+\underline{W}_{0}\right) / 2$, $W_{+}=\left(\bar{W}_{0}-\underline{W}_{0}\right) / 2, W^{*}=\left(\bar{W}_{1}+\underline{W}_{1}\right) / 2, W_{*}=\left(\bar{W}_{1}-\right.$ $\left.\underline{W}_{1}\right) / 2, B_{k}^{+}=\left(\bar{B}_{k}+\underline{B}_{k}\right) / 2$, and $B_{k+}=\left(\bar{B}_{k}-\underline{B}_{k}\right) / 2$, $k=1, \ldots, n$. Let $I$ and 0 denote the identity matrix and the zero matrix with appropriate dimensions, respectively.

Definition 2.1: If there exist positive constants $k>0$ and $\gamma \geq 1$ such that $\|u(t)\| \leq \gamma e^{-k t} \sup _{-\rho \leq \varsigma \leq 0}\|u(\varsigma)\|, \forall t \geq 0$, then the origin is said to be globally exponentially stable for system (1) with $U=0$, where $u(t)=\left(u_{1}(t), \ldots, u_{n}(t)\right)^{T}$ and $k$ is called the exponential convergence rate.

Assumption 2.1: For any $\xi, \zeta \in \Re$ and $\xi \neq \zeta$, the activation functions $\bar{g}_{i}(\cdot)$ and $\bar{f}_{i}(\cdot)$ satisfy the following conditions:

$$
\begin{aligned}
& 0 \leq \frac{\bar{g}_{i}(\xi)-\bar{g}_{i}(\zeta)}{\xi-\zeta} \leq \delta_{i}^{g} \\
& 0 \leq \frac{\bar{f}_{i}(\xi)-\bar{f}_{i}(\zeta)}{\xi-\zeta} \leq \delta_{i}^{f}
\end{aligned}
$$

where $\delta_{i}^{g}>0$ and $\delta_{i}^{f}>0$ are constants, $i=1, \ldots, n$.

Assumption 2.2: Function $a_{i}\left(u_{i}(t)\right)$ satisfies

$$
\frac{a_{i}(\xi)-a_{i}(\zeta)}{\xi-\zeta} \geq \gamma_{i}>0
$$

$\forall \xi, \zeta \in \Re$ and $\xi \neq \zeta, i=1, \ldots, n$.

Assumption 2.3: Amplification function $d_{i}\left(u_{i}(t)\right)$ is positive, continuous, and bounded, i.e., $0<\underline{d}_{i} \leq d_{i}\left(u_{i}(t)\right) \leq \bar{d}_{i}<\infty$, $\underline{d}=\min _{1 \leq i \leq n}\left(\underline{d}_{i}\right), \bar{d}=\max _{1 \leq i \leq n}\left(\bar{d}_{i}\right), i=1, \ldots, n$.

Let $\Delta_{g}=\operatorname{diag}\left(\delta_{1}^{g}, \ldots, \delta_{n}^{g}\right), \Delta_{f}=\operatorname{diag}\left(\delta_{1}^{f}, \ldots, \delta_{n}^{f}\right), \Gamma=$ $\operatorname{diag}\left(\gamma_{1}, \ldots, \gamma_{n}\right)$, and $\Gamma_{m}=\min _{1 \leq i \leq n}\left(\gamma_{i}\right)$.

Lemma 2.1 (cf., [7] and [48]): Let $a$ and $b$ be constants with $0<b<a, \rho \geq 0$. Let $y(t)$ be a continuous function that is nonnegative on $\left[t_{0}-\rho, t_{0}\right]$ and that satisfies the following inequality:

$$
\frac{d y(t)}{d t} \leq-a y(t)+b \bar{y}(t)
$$

for $t \geq t_{0}$, where $\bar{y}(t)=\sup _{t-\rho \leq s \leq t} y(s)$. Then, when $t \geq t_{0}$, we have $y(t) \leq \bar{y}\left(t_{0}\right) e^{-k\left(t-t_{0}\right)}$, where $k$ is the unique positive solution of the equation $k=a-b e^{k \rho}$.

Lemma 2.2 (cf., [23] and [38]): For two vectors $x$ and $y$, a constant $\beta>0$, and a matrix $P>0$ with appropriate dimension, the following inequality holds:

$$
2 x^{T} y \leq \beta x^{T} P x+\beta^{-1} y^{T} P^{-1} y .
$$


Lemma 2.3 (cf., [5] and [8]): For any matrix $A \in[\underline{A}, \bar{A}]$, the following inequality holds:

$$
\|A\| \leq\left\|A^{+}\right\|+\left\|A_{+}\right\|
$$

where $A^{+}=(\bar{A}+\underline{A}) / 2$ and $A_{+}=(\bar{A}-\underline{A}) / 2$.

Lemma 2.4: For two vectors $x$ and $y$, a constant $\alpha>0$, and matrices $P>0$ and $W_{a} \in\left[\underline{W}_{a}, \bar{W}_{a}\right]$ with appropriate dimensions, the following inequalities hold:

$$
\begin{aligned}
& 2 x^{T} P W_{a} y \leq \alpha^{-1} x^{T} P P x+\alpha y^{T}\left(\left\|W_{a}^{+}\right\|+\left\|W_{a_{+}}\right\|\right)^{2} y \\
& 2 x^{T} P W_{a} y \leq \alpha^{-1} x^{T} P\left(\left\|W_{a}^{+}\right\|+\left\|W_{a_{+}}\right\|\right)^{2} P x+\alpha y^{T} y
\end{aligned}
$$

where $W_{a}^{+}=\left(\bar{W}_{a}+\underline{W}_{a}\right) / 2$ and $W_{a_{+}}=\left(\bar{W}_{a}-\underline{W}_{a}\right) / 2$.

Proof: By Lemmas 2.2 and 2.3, we have

$$
\begin{aligned}
& 2 x^{T} P W_{a} y \\
& \leq \alpha^{-1} x^{T} P P x+\alpha y^{T} \\
& \quad \times\left[\left(W_{a}^{T} W_{a}-\left\|W_{a}\right\|^{2}\right)+\left\|W_{a}\right\|^{2}\right. \\
& \left.\quad \quad-\left(\left\|W_{a}^{+}\right\|+\left\|W_{a_{+}}\right\|\right)^{2}+\left(\left\|W_{a}^{+}\right\|+\left\|W_{a_{+}}\right\|\right)^{2}\right] y \\
& \leq \alpha^{-1} x^{T} P P x+\alpha y^{T}\left(\left\|W_{a}^{+}\right\|+\left\|W_{a_{+}}\right\|\right)^{2} y
\end{aligned}
$$

which means that inequality (5) holds. Similarly

$$
\begin{aligned}
& 2 x^{T} P W_{a} y \\
& \leq \alpha^{-1} x^{T} P\left[\left(W_{a} W_{a}^{T}-\left\|W_{a}\right\|^{2}\right)+\left\|W_{a}\right\|^{2}\right. \\
& \left.-\left(\left\|W_{a}^{+}\right\|+\left\|W_{a_{+}}\right\|\right)^{2}+\left(\left\|W_{a}^{+}\right\|+\left\|W_{a_{+}}\right\|\right)^{2}\right] \\
& \times P x+\alpha y^{T} y \\
& \leq \alpha^{-1} x^{T} P\left(\left\|W_{a}^{+}\right\|+\left\|W_{a_{+}}\right\|\right)^{2} P x+\alpha y^{T} y \text {. }
\end{aligned}
$$

\section{Global Robust ExPONENTIAL Stability RESUlts}

\section{A. Cohen-Grossberg Neural Networks With Multiple Time-Varying Delays}

Let $u^{*}=\left(u_{1}^{*}, \ldots, u_{n}^{*}\right)^{T}$ be an equilibrium point of system (1). By coordinate transformation $x_{i}=u_{i}-u_{i}^{*}$, and using the same method as that in [45], we get the following system:

$$
\dot{x}=-D(x)\left[A(x)-W_{0} g(x)-\sum_{i=1}^{n} B_{i} f\left(x\left(t-\bar{\tau}_{i}(t)\right)\right)\right]
$$

where $x(t)=\left(x_{1}(t), \ldots, x_{n}(t)\right)^{T}, D(x)=\operatorname{diag}\left(D_{1}\left(x_{1}\right)\right.$, $\left.\ldots, D_{n}\left(x_{n}\right)\right), D_{i}\left(x_{i}\right)=d_{i}\left(x_{i}+u_{i}^{*}\right), A(x)=\left(A_{1}\left(x_{1}\right)\right.$, $\left.\ldots, A_{n}\left(x_{n}\right)\right)^{T}, A_{i}\left(x_{i}\right)=a_{i}\left(x_{i}+u_{i}^{*}\right)-a_{i}\left(u_{i}^{*}\right), g(x)=$ $\left(g_{1}\left(x_{1}\right), \ldots, g_{n}\left(x_{n}\right)\right)^{T}, g_{i}\left(x_{i}\right)=\bar{g}_{i}\left(x_{i}+u_{i}^{*}\right)-\bar{g}_{i}\left(u_{i}^{*}\right), f(x(t-$ $\left.\left.\bar{\tau}_{i}(t)\right)\right)=\left(f_{1}\left(x_{1}\left(t-\tau_{i 1}(t)\right)\right), \ldots, f_{n}\left(x_{n}\left(t-\tau_{i n}(t)\right)\right)\right)^{T}$, $f_{j}\left(x_{j}\left(t-\tau_{i j}(t)\right)\right)=\bar{f}_{j}\left(x_{j}\left(t-\tau_{i j}(t)\right)+u_{j}^{*}\right)-\bar{f}_{j}\left(u_{j}^{*}\right)$, and $\bar{\tau}_{i}(t)=\left(\tau_{i 1}(t), \ldots, \tau_{i n}(t)\right)^{T}$ for $i, j=1, \ldots, n$.
According to Assumption 2.1, we have $0 \leq g_{i}\left(x_{i}\right) / x_{i} \leq \delta_{i}^{g}$ and $0 \leq f_{i}\left(x_{i}\right) / x_{i} \leq \delta_{i}^{f}$ for $\forall x_{i} \neq 0$. By Assumption 2.2, we have $x_{i} A_{i}\left(x_{i}\right) \geq \gamma_{i} x_{i}^{2}, i=1, \ldots, n$.

Obviously, the problem of global exponential stability of the equilibrium point $u^{*}$ of system (1) is equivalent to the problem of global exponential stability of the origin of system (7).

Theorem 3.1: Under Assumptions 2.1-2.3, if there exist positive-definite diagonal matrices $P, Q, M, R_{i}$, and $S_{i}$, and constants $\theta>0, \alpha>0, \beta_{i}>0, \beta>0, \zeta_{i}>0, \varepsilon_{i}>0$, and $\varepsilon>0$, $i=1, \ldots, n$, such that the following matrix inequalities hold:

$$
\begin{aligned}
& \Omega_{w}=\left[\begin{array}{ccc}
\Phi_{a} & \sum_{i=1}^{n} R_{i} \Delta_{g}-Q \Gamma_{m} & \Phi_{b} \\
* & \Phi_{c} & 0 \\
* & * & \Phi_{d}
\end{array}\right]<0 \\
& \frac{P}{\bar{d}}>\sum_{i=1}^{n}\left(\zeta_{i}+\beta_{i}+\varepsilon_{i}\right) \Delta_{f} \Delta_{f}
\end{aligned}
$$

then the equilibrium point of system (7) is unique, and it is globally robustly exponentially stable, independent of time-varying delays, where

$$
\begin{aligned}
\Phi_{a}= & -2 P \Gamma_{m}+\frac{\left(P+Q \Delta_{g}+M \Delta_{f}\right)}{\underline{d}}+\theta I+\alpha^{-1} P P \\
& +\sum_{i=1}^{n} \beta_{i}^{-1} P\left(\left\|B_{i+}\right\|+\left\|B_{i}^{+}\right\|\right)^{2} P \\
\Phi_{b}= & \sum_{i=1}^{n} S_{i} \Delta_{f}-M \Gamma_{m} \\
\Phi_{c}= & \beta^{-1} Q\left(\left\|W_{+}\right\|+\left\|W^{+}\right\|\right)^{2} Q+\beta I \\
& +\alpha\left(\left\|W_{+}\right\|+\left\|W^{+}\right\|\right)^{2} I \\
& +\sum_{i=1}^{n} \zeta_{i}^{-1} Q\left(\left\|B_{i+}\right\|+\left\|B_{i}^{+}\right\|\right)^{2} Q \\
& +\varepsilon\left(\left\|W_{+}\right\|+\left\|W^{+}\right\|\right)^{2} I-\sum_{i=1}^{n} 2 R_{i} \\
\Phi_{d}= & \sum_{i=1}^{n} \varepsilon_{i}^{-1} M\left(\left\|B_{i+}\right\|+\left\|B_{i}^{+}\right\|\right)^{2} M+\varepsilon^{-1} M M-\sum_{i=1}^{n} 2 S_{i}
\end{aligned}
$$

and $*$ corresponds to the symmetric part in a matrix.

Proof: We will prove the theorem in two steps. First, we will show that (8) and (9) are sufficient conditions guaranteeing the uniqueness of the equilibrium point of system (7). Consider (7) at equilibrium point $x^{*}$, i.e.,

$$
0=-A\left(x^{*}\right)+W_{0} g\left(x^{*}\right)+\sum_{i=1}^{n} B_{i} f\left(x^{*}\right) .
$$

If $g\left(x^{*}\right)=0$ and $f\left(x^{*}\right)=0$, then it is easy to see $x^{*}=0$. Now suppose $x^{*} \neq 0, g\left(x^{*}\right) \neq 0$, and $f\left(x^{*}\right) \neq 0$. Multiplying $2 x^{* T} P$ and $2 g^{T}\left(x^{*}\right) Q, 2 f^{T}\left(x^{*}\right) M$ on both sides of (10), respectively, we have

$$
0=2 x^{* T} P\left[-A\left(x^{*}\right)+W_{0} g\left(x^{*}\right)+\sum_{i=1}^{n} B_{i} f\left(x^{*}\right)\right]
$$




$$
\begin{aligned}
& +2 g^{T}\left(x^{*}\right) Q\left[-A\left(x^{*}\right)+W_{0} g\left(x^{*}\right)+\sum_{i=1}^{n} B_{i} f\left(x^{*}\right)\right] \\
& +2 f^{T}\left(x^{*}\right) M\left[-A\left(x^{*}\right)+W_{0} g\left(x^{*}\right)+\sum_{i=1}^{n} B_{i} f\left(x^{*}\right)\right] \\
& \leq-2 x^{* T} P \Gamma_{m} x^{*}+2 x^{* T} P\left[W_{0} g\left(x^{*}\right)+\sum_{i=1}^{n} B_{i} f\left(x^{*}\right)\right] \\
& -2 g^{T}\left(x^{*}\right) Q \Gamma_{m} x^{*}+2 g^{T}\left(x^{*}\right) Q \\
& \times\left[W_{0} g\left(x^{*}\right)+\sum_{i=1}^{n} B_{i} f\left(x^{*}\right)\right]-2 f^{T}\left(x^{*}\right) M \Gamma_{m} x^{*} \\
& +2 f^{T}\left(x^{*}\right) M\left[W_{0} g\left(x^{*}\right)+\sum_{i=1}^{n} B_{i} f\left(x^{*}\right)\right] .
\end{aligned}
$$

Note that the following inequalities hold for positive-definite diagonal matrices $R_{i}>0$ and $S_{i}>0, i=1, \ldots, n$,:

$$
\begin{aligned}
& 0 \leq 2 g^{T}\left(x^{*}\right) R_{i} \Delta_{g} x^{*}-2 g^{T}\left(x^{*}\right) R_{i} g\left(x^{*}\right) \\
& 0 \leq 2 f^{T}\left(x^{*}\right) S_{i} \Delta_{f} x^{*}-2 f^{T}\left(x^{*}\right) S_{i} f\left(x^{*}\right) .
\end{aligned}
$$

By Lemmas 2.2-2.4 and the above two inequalities, from (11), we have

$$
\begin{aligned}
0 \leq & -2 x^{* T} P \Gamma_{m} x^{*}+\alpha^{-1} x^{* T} P P x^{*} \\
& +\alpha g^{T}\left(x^{*}\right)\left(\left\|W_{+}\right\|+\left\|W^{+}\right\|\right)^{2} g\left(x^{*}\right) \\
& +\sum_{i=1}^{n} \beta_{i}^{-1} x^{* T} P\left(\left\|B_{i+}\right\|+\left\|B_{i}^{+}\right\|\right)^{2} P x^{*} \\
& +\sum_{i=1}^{n} \beta_{i} f^{T}\left(x^{*}\right) f\left(x^{*}\right)-2 g^{T}\left(x^{*}\right) Q \Gamma_{m} x^{*} \\
& +g^{T}\left(x^{*}\right)\left[\beta^{-1} Q\left(\left\|W_{+}\right\|+\left\|W^{+}\right\|\right)^{2} Q+\beta I\right] g\left(x^{*}\right) \\
& +\sum_{i=1}^{n} \zeta_{i}^{-1} g^{T}\left(x^{*}\right) Q\left(\left\|B_{i+}\right\|+\left\|B_{i}^{+}\right\|\right)^{2} Q g\left(x^{*}\right) \\
& +\sum_{i=1}^{n} \zeta_{i} f^{T}\left(x^{*}\right) f\left(x^{*}\right)-2 f^{T}\left(x^{*}\right) M \Gamma_{m} x^{*} \\
& +\varepsilon^{-1} f^{T}\left(x^{*}\right) M M f\left(x^{*}\right) \\
& +\varepsilon g^{T}\left(x^{*}\right)\left(\left\|W_{+}\right\|+\left\|W^{+}\right\|\right)^{2} g\left(x^{*}\right) \\
& +\sum_{i=1}^{n} \varepsilon_{i}^{-1} f^{T}\left(x^{*}\right) M\left(\left\|B_{i+}\right\|+\left\|B_{i}^{+}\right\|\right)^{2} M f\left(x^{*}\right) \\
& +\sum_{i=1}^{n} \varepsilon_{i} f^{T}\left(x^{*}\right) f\left(x^{*}\right) \\
& +\sum_{i=1}^{n}\left[2 g^{T}\left(x^{*}\right) R_{i} \Delta_{g} x^{*}-2 g^{T}\left(x^{*}\right) R_{i} g\left(x^{*}\right)\right] \\
& +\sum_{i=1}^{n}\left[2 f^{T}\left(x^{*}\right) S_{i} \Delta_{f} x^{*}-2 f^{T}\left(x^{*}\right) S_{i} f\left(x^{*}\right)\right] \\
& +x^{* T}\left[\frac{\left(P+Q \Delta_{g}+M \Delta_{f}\right)}{\underline{d}}+\theta I\right] x^{*}-x^{* T} P \frac{x^{*}}{\bar{d}}
\end{aligned}
$$

By (9), we have

$$
\begin{aligned}
& \sum_{i=1}^{n}\left(\beta_{i}+\zeta_{i}+\varepsilon_{i}\right) f^{T}\left(x^{*}\right) f\left(x^{*}\right)-\frac{x^{* T} P x^{*}}{\bar{d}} \\
& \quad \leq \sum_{i=1}^{n}\left(\beta_{i}+\zeta_{i}+\varepsilon_{i}\right) x^{* T} \Delta_{f} \Delta_{f} x^{*}-\frac{x^{* T} P x^{*}}{\bar{d}} \\
& \quad<0 .
\end{aligned}
$$

Substituting (13) into (12) and after rearranging, we have

$$
0 \leq\left[\begin{array}{llll}
x^{* T} & g^{T}\left(x^{*}\right) & f^{T}\left(x^{*}\right)
\end{array}\right] \Omega_{w}\left[\begin{array}{lll}
x^{* T} & g^{T}\left(x^{*}\right) & f^{T}\left(x^{*}\right)
\end{array}\right]^{T}
$$

where $\Omega_{w}$ is defined in (8). However, for $g\left(x^{*}\right) \neq 0, f\left(x^{*}\right) \neq 0$, and $x^{*} \neq 0$, from (8), we get

$$
\left[\begin{array}{lll}
x^{* T} & g^{T}\left(x^{*}\right) & f^{T}\left(x^{*}\right)
\end{array}\right] \Omega_{w}\left[\begin{array}{lll}
x^{* T} & g^{T}\left(x^{*}\right) & f^{T}\left(x^{*}\right)
\end{array}\right]^{T}<0 .
$$

Obviously, (15) contradicts with (14), which in turn implies that at the equilibrium point $x^{*}, f\left(x^{*}\right)=0$ and $g\left(x^{*}\right)=0$. This means that the origin of (7) is a unique equilibrium point for a given $U$.

Second, we will show that conditions (8) and (9) are also sufficient conditions guaranteeing the global robust exponential stability of the equilibrium point of system (7). Choose the following Lyapunov functional:

$$
\begin{aligned}
V_{0}(x)= & 2 \sum_{i=1}^{n} p_{i} \int_{0}^{x_{i}(t)} \frac{s}{D_{i}(s)} d s+2 \sum_{i=1}^{n} q_{i} \int_{0}^{x_{i}(t)} \frac{g_{i}(s)}{D_{i}(s)} d s \\
& +2 \sum_{i=1}^{n} m_{i} \int_{0}^{x_{i}(t)} \frac{f_{i}(s)}{D_{i}(s)} d s
\end{aligned}
$$

where $p_{i}, q_{i}$, and $m_{i}$ are all positive numbers. The derivative of $V_{0}(x)$ along the trajectories of (7) is as follows:

$$
\begin{aligned}
\dot{V}_{0}(x)= & 2 x^{T} P\left[-A(x)+W_{0} g(x)+\sum_{i=1}^{n} B_{i} f\left(x\left(t-\bar{\tau}_{i}(t)\right)\right)\right] \\
& +2 g^{T}(x) Q\left[-A(x)+W_{0} g(x)+\sum_{i=1}^{n} B_{i} f\left(x\left(t-\bar{\tau}_{i}(t)\right)\right)\right] \\
& -2 f^{T}(x) M A(x) \\
& +2 f^{T}(x) M\left[W_{0} g(x)+\sum_{i=1}^{n} B_{i} f\left(x\left(t-\bar{\tau}_{i}(t)\right)\right)\right]
\end{aligned}
$$

where $P=\operatorname{diag}\left(p_{1}, \ldots, p_{n}\right), Q=\operatorname{diag}\left(q_{1}, \ldots, q_{n}\right)$ and $M=$ $\operatorname{diag}\left(m_{1}, \ldots, m_{n}\right)$.

Using Assumption 2.1 and Lemmas 2.2-2.4 again, (17) becomes

$$
\begin{aligned}
\dot{V}_{0}(x) \leq & -2 x^{T} P \Gamma_{m} x+\alpha g^{T}(x)\left(\left\|W_{+}\right\|+\left\|W^{+}\right\|\right)^{2} g(x) \\
& +\sum_{i=1}^{n} \beta_{i}^{-1} x^{T} P\left(\left\|B_{i+}\right\|+\left\|B_{i}^{+}\right\|\right)^{2} P x \\
& +\sum_{i=1}^{n} \beta_{i} f^{T}\left(x\left(t-\bar{\tau}_{i}(t)\right)\right) f\left(x\left(t-\bar{\tau}_{i}(t)\right)\right)
\end{aligned}
$$




$$
\begin{aligned}
& +\alpha^{-1} x^{T} P P x-2 g^{T}(x) Q \Gamma_{m} x \\
& +g^{T}(x)\left[\beta^{-1} Q\left(\left\|W_{+}\right\|+\left\|W^{+}\right\|\right)^{2} Q+\beta I\right] g(x) \\
& +\sum_{i=1}^{n} \zeta_{i}^{-1} g^{T}(x) Q\left(\left\|B_{i+}\right\|+\left\|B_{i}^{+}\right\|\right)^{2} Q g(x) \\
& +\sum_{i=1}^{n} \zeta_{i} f^{T}\left(x\left(t-\bar{\tau}_{i}(t)\right)\right) f\left(x\left(t-\bar{\tau}_{i}(t)\right)\right) \\
& -2 f^{T}(x) M \Gamma_{m} x+\varepsilon^{-1} f^{T}(x) M M f(x) \\
& +\varepsilon g^{T}(x)\left(\left\|W_{+}\right\|+\left\|W^{+}\right\|\right)^{2} g(x) \\
& +\sum_{i=1}^{n} \varepsilon_{i}^{-1} f^{T}(x) M\left(\left\|B_{i+}\right\|+\left\|B_{i}^{+}\right\|\right)^{2} M f(x) \\
& +\sum_{i=1}^{n} \varepsilon_{i} f^{T}\left(x\left(t-\bar{\tau}_{i}(t)\right)\right) f\left(x\left(t-\bar{\tau}_{i}(t)\right)\right) \\
& +\sum_{i=1}^{n} 2 f^{T}(x) S_{i} \Delta_{f} x-\sum_{i=1}^{n} 2 f^{T}(x) S_{i} f(x) \\
& +\sum_{i=1}^{n} 2 g^{T}(x) R_{i} \Delta_{g} x-\sum_{i=1}^{n} 2 g^{T}(x) R_{i} g(x) \\
& +x^{T}\left[\frac{\left(P+Q \Delta_{g}+M \Delta_{f}\right)}{\underline{d}}+\theta I\right] x \\
& -x^{T}\left[\frac{\left(P+Q \Delta_{g}+M \Delta_{f}\right)}{\underline{d}}+\theta I\right] x \\
& \leq\left[\begin{array}{lll}
x^{T} & g^{T}(x) & f^{T}(x)
\end{array}\right] \Omega_{w}\left[\begin{array}{lll}
x^{T} & g^{T}(x) & f^{T}(x)
\end{array}\right]^{T} \\
& -x^{T}\left[\frac{\left(P+Q \Delta_{g}+M \Delta_{f}\right)}{\underline{d}}+\theta I\right] x \\
& +\sum_{i=1}^{n}\left(\beta_{i}+\zeta_{i}+\varepsilon_{i}\right) x\left(t-\bar{\tau}_{i}(t)\right)^{T} \Delta_{f} \Delta_{f} x\left(t-\bar{\tau}_{i}(t)\right) .
\end{aligned}
$$

From the Lyapunov functional (16), we have

$$
V_{0}(x) \leq \frac{1}{\underline{d}} x^{T}\left(P+Q \Delta_{g}+M \Delta_{f}\right) x .
$$

Considering (9), we have

$$
\sum_{i=1}^{n}\left(\beta_{i}+\zeta_{i}+\varepsilon_{i}\right) x\left(t-\bar{\tau}_{i}(t)\right)^{T} \Delta_{f} \Delta_{f} x\left(t-\bar{\tau}_{i}(t)\right) \leq \bar{V}_{0}(x)
$$

where $\bar{V}_{0}(x)=\sup _{t-\rho \leq s \leq t} V_{0}(x(s))$.

Because $\left(P+Q \Delta_{g}+\bar{M} \bar{\Delta}_{f}\right) / \underline{d}+\theta I>\left(P+Q \Delta_{g}+M \Delta_{f}\right) / \underline{d}$, there exists a constant $\eta_{0}>1$ such that $x^{T}\left[\left(P+Q \Delta_{g}+\right.\right.$ $\left.\left.M \Delta_{f}\right) / \underline{d}+\theta I\right] x \geq \eta_{0} x^{T}\left[\left(P+Q \Delta_{g}+M \Delta_{f}\right) / \underline{d}\right] x \geq \eta_{0} V_{0}(x)$ for $x \neq 0$. Therefore, combining (19) with (20), (18) becomes

$$
\dot{V}_{0}(x) \leq-\eta_{0} V_{0}(x)+\bar{V}_{0}(x)
$$

where $1<\eta_{0} \leq \lambda_{m}\left(I+\theta \underline{d}\left(P+Q \Delta_{g}+M \Delta_{f}\right)^{-1}\right)$.

By Lemma $2.1, V_{0}(x) \leq \bar{V}_{0}(x(0)) e^{-k t} \leq(1 / \underline{d}) \lambda_{M}(P+$ $\left.Q \Delta_{g}+M \Delta_{f}\right) e^{-k t} \sup _{t-\rho \leq s \leq t}\|x(s)\|^{2}$, where $k=\eta_{0}-e^{k \rho}$. Furthermore, from (16), we have $\lambda_{m}(P) x^{T} x / \bar{d} \leq V_{0}(x)$, which leads to

$$
\|x(t)\| \leq \sqrt{\frac{\bar{d} \lambda_{M}\left(P+Q \Delta_{g}+M \Delta_{f}\right)}{\underline{d} \lambda_{m}(P)}} e^{-(k t / 2)} \sup _{-\rho \leq s \leq 0}\|x(s)\| .
$$

By Definition 2.1, the equilibrium point of (7) is globally robustly exponentially stable if conditions (8) and (9) hold.

When $\bar{g}_{j}(\cdot)=\bar{f}_{j}(\cdot)$ in $(1), j=1, \ldots, n$, the model (7) becomes

$$
\dot{x}=-D(x)\left[A(x)-W_{0} f(x)-\sum_{i=1}^{n} B_{i} f\left(x\left(t-\bar{\tau}_{i}(t)\right)\right)\right]_{(22)}
$$

In a similar procedure to the proof of Theorem 3.1, we have the following result.

Corollary 3.1: Under Assumptions 2.1-2.3, if there exist positive-definite diagonal matrices $P, Q$, and $R$, and constants $\theta>$ $0, \alpha>0, \beta_{i}>0, \beta>0$, and $\zeta_{i}>0$ such that the following matrix inequalities hold:

$$
\begin{aligned}
\Omega_{w}^{0} & =\left[\begin{array}{cc}
\Phi_{e} & R \Delta-Q \Gamma_{m} \\
R \Delta-Q \Gamma_{m} & \Phi_{f}
\end{array}\right]<0 \\
\overline{\bar{d}} & >\sum_{i=1}^{n}\left(\zeta_{i}+\beta_{i}\right) \Delta \Delta
\end{aligned}
$$

then the equilibrium point of system (22) is unique, and it is globally robustly exponentially stable, independent of time-varying delays, where $\Delta=\Delta_{g}=\Delta_{f}$

$$
\begin{aligned}
\Phi_{e}= & -2 P \Gamma_{m}+\frac{(P+Q \Delta)}{\underline{d}}+\theta I \\
& +\alpha^{-1} P\left(\left\|W_{+}\right\|+\left\|W^{+}\right\|\right)^{2} P \\
& +\sum_{i=1}^{n} \beta_{i}^{-1} P\left(\left\|B_{i+}\right\|+\left\|B_{i}^{+}\right\|\right)^{2} P \\
\Phi_{f}= & \beta^{-1} Q\left(\left\|W_{+}\right\|+\left\|W^{+}\right\|\right)^{2} Q+\beta I+\alpha I \\
& +\sum_{i=1}^{n} \zeta_{i}^{-1} Q\left(\left\|B_{i+}\right\|+\left\|B_{i}^{+}\right\|\right)^{2} Q-2 R .
\end{aligned}
$$

Proof: The uniqueness of the equilibrium point of system (22) can be proved similarly to the proof of Theorem 3.1. The details are omitted here.

Next, we will show that conditions (23) and (24) are also sufficient conditions guaranteeing the global robust exponential stability of the equilibrium point of system (22). Choose the following Lyapunov functional:

$$
V_{1}(x)=2 \sum_{i=1}^{n} p_{i} \int_{0}^{x_{i}(t)} \frac{s}{D_{i}(s)} d s+2 \sum_{i=1}^{n} q_{i} \int_{0}^{x_{i}(t)} \frac{f_{i}(s)}{D_{i}(s)} d s
$$

where $p_{i}$ and $q_{i}$ are all positive numbers. The derivative of $V_{1}(x)$ along the trajectories of (22) is as follows:

$$
\begin{aligned}
\dot{V}_{1}(x)= & 2 x^{T} P\left[-A(x)+W_{0} f(x)+\sum_{i=1}^{n} B_{i} f\left(x\left(t-\bar{\tau}_{i}(t)\right)\right)\right] \\
& +2 f^{T}(x) Q\left[-A(x)+W_{0} f(x)+\sum_{i=1}^{n} B_{i} f\left(x\left(t-\bar{\tau}_{i}(t)\right)\right)\right]
\end{aligned}
$$

where $P=\operatorname{diag}\left(p_{1}, \ldots, p_{n}\right)$ and $Q=\operatorname{diag}\left(q_{1}, \ldots, q_{n}\right)$.

Using Assumption 2.1 and Lemmas 2.2-2.4 again, (26) becomes

$$
\begin{aligned}
\dot{V}_{1}(x) \leq & -2 x^{T} P \Gamma_{m} x+\alpha^{-1} x^{T} P\left(\left\|W_{+}\right\|+\left\|W^{+}\right\|\right)^{2} P x \\
& +\alpha f^{T}(x) f(x)-2 f^{T}(x) Q \Gamma_{m} x
\end{aligned}
$$




$$
\begin{aligned}
& +\sum_{i=1}^{n} \beta_{i}^{-1} x^{T} P\left(\left\|B_{i+}\right\|+\left\|B_{i}^{+}\right\|\right)^{2} P x \\
& +\sum_{i=1}^{n} \beta_{i} f^{T}\left(x\left(t-\bar{\tau}_{i}(t)\right)\right) f\left(x\left(t-\bar{\tau}_{i}(t)\right)\right) \\
& +f^{T}(x)\left[\beta^{-1} Q\left(\left\|W_{+}\right\|+\left\|W^{+}\right\|\right)^{2} Q+\beta I\right] f(x) \\
& +\sum_{i=1}^{n} \zeta_{i}^{-1} f^{T}(x) Q\left(\left\|B_{i+}\right\|+\left\|B_{i}^{+}\right\|\right)^{2} Q f(x) \\
& +\sum_{i=1}^{n} \zeta_{i} f^{T}\left(x\left(t-\bar{\tau}_{i}(t)\right)\right) f\left(x\left(t-\bar{\tau}_{i}(t)\right)\right) \\
& +2 f^{T}(x) R \Delta x-2 f^{T}(x) R f(x) \\
& +x^{T}\left[\frac{(P+Q \Delta)}{\underline{d}}+\theta I\right] x-x^{T}\left[\frac{(P+Q \Delta)}{\underline{d}}+\theta I\right] x \\
& \leq \\
& {\left[x^{T} \quad f^{T}(x)\right] \Omega_{w}^{0}\left[x^{T} \quad f^{T}(x)\right]^{T}} \\
& \quad-x^{T}\left[\frac{(P+Q \Delta)}{\underline{d}}+\theta I\right] x \\
& +\sum_{i=1}^{n}\left(\beta_{i}+\zeta_{i}\right) x\left(t-\bar{\tau}_{i}(t)\right)^{T} \Delta \Delta x\left(t-\bar{\tau}_{i}(t)\right) .
\end{aligned}
$$

From the Lyapunov functional (25), we have

$$
V_{1}(x) \leq \frac{1}{\underline{d}} x^{T}(P+Q \Delta) x
$$

Considering (24) and (28), (27) becomes

$$
\dot{V}_{1}(x) \leq-\eta_{1} V_{1}(x)+\bar{V}_{1}(x)
$$

where $1<\eta_{1} \leq \lambda_{m}\left(I+\theta \underline{d}(P+Q \Delta)^{-1}\right), \bar{V}_{1}(x)=$ $\sup _{t-\rho \leq s \leq t} V_{1}(x(s))$.

Therefore, by Lemma 2.1

$$
V_{1}(x) \leq \frac{\lambda_{M}(P+Q \Delta)}{\underline{d}} e^{-k t} \sup _{t-\rho \leq s \leq t}\|x(s)\|^{2}
$$

where $k=\eta_{1}-e^{k \rho}$. Furthermore, from (25), we have $\lambda_{m}(P) x^{T} x / \bar{d} \leq V_{1}(x) \leq \lambda_{M}(P+Q \Delta) x^{T} x / \underline{d}$, which leads to

$$
\|x(t)\| \leq \sqrt{\frac{\bar{d} \lambda_{M}(P+Q \Delta)}{\underline{d} \lambda_{m}(P)}} e^{-(k t / 2)} \sup _{-\rho \leq s \leq 0}\|x(s)\| .
$$

By Definition 2.1, the equilibrium point of (22) is globally robustly exponentially stable if conditions (23) and (24) hold.

For the case of no uncertainties, we have the following results.

Corollary 3.2: Under Assumptions 2.1-2.3, if there exist positive-definite diagonal matrices $P, Q, M, R_{i}, S_{i}, H_{i}, K_{i}$, and $L_{i}$, and constant $\theta>0, i=1, \ldots, n$, such that the following matrix inequalities hold:

$$
\begin{aligned}
\Omega_{w}^{1} & =\left[\begin{array}{ccc}
\Phi_{a}^{1} & \sum_{i=1}^{n} R_{i} \Delta_{g}-Q \Gamma+P W_{0} & \Phi_{b}^{1} \\
* & \Phi_{c}^{1} & W_{0}^{T} M \\
* & * & \Phi_{d}^{1}
\end{array}\right]<0 \\
\frac{P}{\bar{d}}>\sum_{i=1}^{n} \Delta_{f}\left(H_{i}+K_{i}+L_{i}\right) \Delta_{f} &
\end{aligned}
$$

then the equilibrium point of system (7) without uncertainties is unique, and it is globally exponentially stable, independent of time-varying delays, where

$$
\begin{aligned}
\Phi_{a}^{1}= & -2 P \Gamma+\frac{\left(P+Q \Delta_{g}+M \Delta_{f}\right)}{\underline{d}}+\theta I \\
& \quad+\sum_{i=1}^{n} P B_{i} H_{i}^{-1} B_{i}^{T} P \\
\Phi_{b}^{1}= & \sum_{i=1}^{n} S_{i} \Delta_{f}-M \Gamma \\
\Phi_{c}^{1}= & Q W_{0}+W_{0}^{T} Q+\sum_{i=1}^{n} Q B_{i} K_{i}^{-1} B_{i}^{T} Q-\sum_{i=1}^{n} 2 R_{i} \\
\Phi_{d}^{1}= & \sum_{i=1}^{n} M B_{i} L_{i}^{-1} B_{i}^{T} M-\sum_{i=1}^{n} 2 S_{i}
\end{aligned}
$$

and $*$ corresponds to the symmetric part in a matrix.

Proof: The uniqueness of the equilibrium point of system (7) without uncertainties can be proved similarly to the proof of Theorem 3.1. The details are omitted here.

In the following, we will show that the conditions (30) and (31) are also sufficient conditions guaranteeing the global exponential stability of the equilibrium point of system (7). Choose the same Lyapunov functional $V_{0}(x)$ as that in (16). The derivative of $V_{0}(x)$ along the trajectories of (7) is the same as (17).

Using Assumption 2.1 and Lemma 2.2 again, from (17), we have

$$
\begin{aligned}
\dot{V}_{0}(x) \leq & -2 x^{T} P \Gamma x+2 x^{T} P W_{0} g(x) \\
& +\sum_{i=1}^{n}\left[x^{T} P B_{i} H_{i}^{-1} B_{i}^{T} P x\right. \\
& \left.\quad+f^{T}\left(x\left(t-\bar{\tau}_{i}(t)\right)\right) H_{i} f\left(x\left(t-\bar{\tau}_{i}(t)\right)\right)\right] \\
& -2 g^{T}(x) Q \Gamma x+2 g^{T}(x) Q W_{0} g(x) \\
& +\sum_{i=1}^{n} g^{T}(x) Q B_{i} K_{i}^{-1} B_{i}^{T} Q g(x) \\
& +\sum_{i=1}^{n} f^{T}\left(x\left(t-\bar{\tau}_{i}(t)\right)\right) K_{i} f\left(x\left(t-\bar{\tau}_{i}(t)\right)\right) \\
& -2 f^{T}(x) M \Gamma x+2 f^{T}(x) M W_{0} g(x) \\
& +\sum_{i=1}^{n} f^{T}(x) M B_{i} L_{i}^{-1} B_{i}^{T} M f(x) \\
& +\sum_{i=1}^{n} f^{T}\left(x\left(t-\bar{\tau}_{i}(t)\right)\right) L_{i} f\left(x\left(t-\bar{\tau}_{i}(t)\right)\right) \\
& +\sum_{i=1}^{n} 2 f^{T}(x) S_{i} \Delta_{f} x-\sum_{i=1}^{n} 2 f^{T}(x) S_{i} f(x) \\
& +\sum_{i=1}^{n} 2 g^{T}(x) R_{i} \Delta_{g} x-\sum_{i=1}^{n} 2 g^{T}(x) R_{i} g(x) \\
\leq & -\eta_{0} V_{0}(x)+\bar{V}_{0}(x)
\end{aligned}
$$

where $\eta_{0}$ and $\bar{V}_{0}(x)$ are the same as those defined in (21). The rest of the proof is similar to the proof of Theorem 3.1. The details are omitted. 
Corollary 3.3: Under Assumptions 2.1-2.3, if there exist positive-definite diagonal matrices $P, Q, R$, and $H_{i}$, and constant $\theta, i=1, \ldots, n$, such that the following LMIs hold:

$$
\begin{aligned}
\Omega_{w}^{2} & =\left[\begin{array}{cccccc}
\Phi_{a}^{2} & \Phi_{b}^{2} & P B_{1} & P B_{2} & \ldots & P B_{n} \\
* & \Phi_{c}^{2} & Q B_{1} & Q B_{2} & \ldots & Q B_{n} \\
* & * & -H_{1} & 0 & \ldots & 0 \\
* & * & * & -H_{2} & \ldots & 0 \\
\vdots & \vdots & \vdots & \vdots & \ddots & \vdots \\
* & * & * & * & \ldots & -H_{n}
\end{array}\right]<0 \\
\frac{P}{\bar{d}}>\sum_{i=1}^{n} \Delta H_{i} \Delta &
\end{aligned}
$$

then the equilibrium point of system (22) without uncertainties is unique, and it is globally exponentially stable, independent of time-varying delays, where $\Delta=\Delta_{g}=\Delta_{f}$

$$
\begin{aligned}
\Phi_{a}^{2} & =-2 P \Gamma+\frac{(P+Q \Delta)}{\underline{d}}+\theta I \\
\Phi_{b}^{2} & =R \Delta-Q \Gamma+P W_{0} \\
\Phi_{c}^{2} & =Q W_{0}+W_{0}^{T} Q-2 R
\end{aligned}
$$

and $*$ corresponds to the symmetric part in a matrix.

Proof: The uniqueness of the equilibrium point of system (22) without uncertainties can be proved similarly to the proof of Theorem 3.1. The details are omitted here.

In the following, we will show that conditions (33) and (34) are also sufficient conditions guaranteeing the global exponential stability of the equilibrium point of system (22). Choose the same Lyapunov functional $V_{1}(x)$ as that in (25). The derivative of $V_{1}(x)$ along the trajectories of (22) is as follows:

$$
\begin{aligned}
\dot{V}_{1}(x) \leq & -2 x^{T} P \Gamma x+2 x^{T} P W_{0} f(x) \\
& +2 x^{T} P \sum_{i=1}^{n} B_{i} f\left(x\left(t-\bar{\tau}_{i}(t)\right)\right) \\
& -2 f^{T}(x) Q \Gamma x+2 f^{T}(x) Q W_{0} f(x) \\
& +2 f^{T}(x) Q \sum_{i=1}^{n} B_{i} f\left(x\left(t-\bar{\tau}_{i}(t)\right)\right) \\
& +2 f^{T}(x) R \Delta x-2 f^{T}(x) R f(x) \\
& +\sum_{i=1}^{n} f^{T}\left(x\left(t-\bar{\tau}_{i}(t)\right)\right) H_{i} f\left(x\left(t-\bar{\tau}_{i}(t)\right)\right) \\
& -\sum_{i=1}^{n} f^{T}\left(x\left(t-\bar{\tau}_{i}(t)\right)\right) H_{i} f\left(x\left(t-\bar{\tau}_{i}(t)\right)\right) \\
\leq & -\eta_{1} V_{1}(x)+\bar{V}_{1}(x)
\end{aligned}
$$

where $\eta_{1}$ and $\bar{V}_{1}(x)$ are defined in (29). The remainder of the proof is the same as the proof of Corollary 3.1. The details are omitted.

Remark 3.1: For the robust stability of interval systems, $M$-matrix as a main approach has been utilized widely in [11], [29], [35], and [47]. To the best of our knowledge, no delay-independent robust stability results based on LMI for interval system (1) and (22) with unknown time-varying delay have been reported in the literature. In this paper, Theorems 3.1 can be changed to the LMI structure and independent of time-varying delay, which can be verified efficiently using the well-known interior point algorithms [4]. Moreover, for the interval Cohen-Grossberg neural networks (1), the robust stability results in [11] require that $\dot{\tau}_{i j}(t) \leq 1, \delta_{i}^{f} \leq 1$, and $\delta_{i}^{g} \leq 1$, while our results have no such restrictions on the value of $\delta_{i}^{f}$ and $\delta_{i}^{g}, i=1, \ldots, n$, and the value of the derivative of the time-varying delays may be relaxed to be greater than 1 .

\section{B. Cohen-Grossberg Neural Networks With Single Time-Varying Delay}

Note that Theorem 3.1 can also be generalized to the model (1) with single delay, i.e.,

$$
\begin{aligned}
\frac{d u_{i}(t)}{d t}=-d_{i}\left(u_{i}(t)\right) & {\left[a_{i}\left(u_{i}(t)\right)-\sum_{j=1}^{n} w_{0 i j} \bar{g}_{j}\left(u_{j}(t)\right)\right.} \\
& \left.-\sum_{j=1}^{n} w_{1 i j} \bar{g}_{j}\left(u_{j}(t-\tau(t))\right)+U_{i}\right]
\end{aligned}
$$

where $0 \leq \tau(t) \leq \rho$ and others are the same as those defined in (1).

However, in this case, the conservativeness of Theorem 3.1 will be increased. To derive some less conservative stability results for model (35), we adopt some different skills in the proof procedure. All these stability results cannot directly be derived from the results in Section III-A.

Let $u^{*}=\left(u_{1}^{*}, \ldots, u_{n}^{*}\right)^{T}$ be an equilibrium point of system (35). By coordinate transformation $x_{i}=u_{i}-u_{i}^{*}$, we get the following system:

$$
\dot{x}=-D(x)\left[A(x)-W_{0} f(x)-W_{1} f(x(t-\tau(t)))\right]
$$

where $f(x)=\left(f_{1}\left(x_{1}\right), \ldots, f_{n}\left(x_{n}\right)\right)^{T}, f_{i}\left(x_{i}\right)=\bar{g}_{i}\left(x_{i}+u_{i}^{*}\right)-$ $\bar{g}_{i}\left(u_{i}^{*}\right)$, and others are the same as those defined in (7). By Assumption 2.1, we have $0 \leq f_{i}\left(x_{i}\right) / x_{i} \leq \delta_{i}^{f}=\delta_{i}$ for $\forall x_{i} \neq 0$. Let $\Delta=\operatorname{diag}\left(\delta_{1}, \ldots, \delta_{n}\right)$ and $\delta_{M}=\max _{1<i<n} \delta_{i}$.

Theorem 3.2: Under Assumptions 2.1-2.3, if there exist positive-definite diagonal matrices $P, Q$, and $R$ and constants $\theta>0$, $\alpha>0, \beta>0, \gamma>0$, and $\varepsilon>0$ such that the following LMIs hold:

$$
\begin{aligned}
& {\left[\begin{array}{cccccc}
\Phi_{11} & \Phi_{12} & 0 & 0 & P & P \\
\Phi_{12}^{T} & \Phi_{22} & Q & Q & 0 & 0 \\
0 & Q & \Phi_{33} & 0 & 0 & 0 \\
0 & Q & 0 & \Phi_{44} & 0 & 0 \\
P & 0 & 0 & 0 & -\alpha I & 0 \\
P & 0 & 0 & 0 & 0 & -\beta I
\end{array}\right]<0} \\
& P \\
& \overline{\bar{d}}>\gamma \Delta^{2}+\beta\left(\left\|W_{*}\right\|+\left\|W^{*}\right\|\right)^{2} \Delta^{2}
\end{aligned}
$$

where $\Phi_{11}=-2 P \Gamma_{m}+(P+Q \Delta) / \underline{d}+\theta I, \Phi_{12}=R \Delta-Q \Gamma_{m}$, $\Phi_{22}=\varepsilon I+\alpha\left(\left\|W_{+}\right\|+\left\|W^{+}\right\|\right)^{2} I-2 R, \Phi_{33}=-\gamma\left(\left\|W_{*}\right\|+\right.$ $\left.\left\|W^{*}\right\|\right)^{-2} I$, and $\Phi_{44}=-\varepsilon\left(\left\|W_{+}\right\|+\left\|W^{+}\right\|\right)^{-2} I$, then the equilibrium point of system (36) is unique, and it is globally robustly exponentially stable, independent of time-varying delay.

Proof: By Schur complement [4], from (37), we have

$$
\Omega=\left[\begin{array}{cc}
\Phi_{1} & R \Delta-Q \Gamma_{m} \\
\left(R \Delta-Q \Gamma_{m}\right)^{T} & \Phi_{2}
\end{array}\right]<0
$$


where $\Phi_{1}=-2 P \Gamma_{m}+(P+Q \Delta) / \underline{d}+\theta I+\alpha^{-1} P P+$ $\beta^{-1} P P$ and $\Phi_{2}=\varepsilon^{-1} Q\left(\left\|W_{+}\right\|+\left\|W^{+}\right\|\right)^{2} Q+\varepsilon I+\alpha\left(\left\|W_{+}\right\|+\right.$ $\left.\left\|W^{+}\right\|\right)^{2} I+\gamma^{-1} Q\left(\left\|W_{*}\right\|+\left\|W^{*}\right\|\right)^{2} Q-2 R$.

Choose the same Lyapunov functional $V_{1}(x)$ as that in (25). The derivative of $V_{1}(x)$ along the trajectories of (36) is as follows:

$$
\begin{aligned}
\dot{V}_{1}(x) \leq & -2 x^{T} P \Gamma_{m} x+2 x^{T} P W_{0} f(x)-2 f^{T}(x) Q \Gamma_{m} x \\
& +2 f^{T}(x) Q W_{0} f(x) \\
& +2\left[f^{T}(x) Q W_{1}+x^{T} P W_{1}\right] f(x(t-\tau(t)))
\end{aligned}
$$

where $P=\operatorname{diag}\left(p_{1}, \ldots, p_{n}\right)$ and $Q=\operatorname{diag}\left(q_{1}, \ldots, q_{n}\right)$.

Using Lemmas 2.2-2.4 and Assumption 2.1, (40) becomes

$$
\begin{aligned}
\dot{V}_{1}(x) \leq & -2 x^{T} P \Gamma_{m} x+\alpha f^{T}(x)\left(\left\|W_{+}\right\|+\left\|W^{+}\right\|\right)^{2} f(x) \\
& +\alpha^{-1} x^{T} P P x-2 f^{T}(x) Q \Gamma_{m} x \\
& +\varepsilon^{-1} f^{T}(x) Q\left(\left\|W_{+}\right\|+\left\|W^{+}\right\|\right)^{2} Q f(x) \\
& +\varepsilon f^{T}(x) f(x)+\beta^{-1} x^{T} P P x \\
& +\beta f^{T}(x(t-\tau(t)))\left(\left\|W_{*}\right\|+\left\|W^{*}\right\|\right)^{2} \\
& \times f(x(t-\tau(t))) \\
& +\gamma^{-1} f^{T}(x) Q\left(\left\|W_{*}\right\|+\left\|W^{*}\right\|\right)^{2} Q f(x) \\
& +\gamma f^{T}(x(t-\tau(t)) f(x(t-\tau(t))) \\
& +2 f^{T}(x) R \Delta x-2 f^{T}(x) R f(x) \\
& +x^{T}\left[\frac{(P+\Delta Q)}{\underline{d}}+\theta I\right] x-x^{T}\left[\frac{(P+\Delta Q)}{\underline{d}}+\theta I\right] x \\
\leq & {\left[x^{T} \quad f^{T}(x)\right] \Omega\left[x^{T} \quad f^{T}(x)\right]^{T} } \\
& -x^{T}\left[\frac{(P+\Delta Q)}{\underline{d}}+\theta I\right] x \\
& +x^{T}(t-\tau(t))\left[\beta\left(\left\|W_{*}\right\|+\left\|W^{*}\right\|\right)^{2}+\gamma\right] \\
& \times \Delta^{2} x(t-\tau(t)) \\
\leq & -\eta_{1} V_{1}(x)+\bar{V}_{1}(x)
\end{aligned}
$$

where $\eta_{1}$ and $\bar{V}_{1}(x)$ are the same as those defined in (29). The rest of the proof is similar to that of Corollary 3.2. The details are omitted.

By choosing another different Lyapunov function

$$
V_{2}(x)=2 \sum_{i=1}^{n} p_{i} \int_{0}^{x_{i}(t)} \frac{s}{D_{i}(s)} d s
$$

where $p_{i}$ are positive numbers. In a similar manner to the proof of Corollary 3.2, we can have the following theorems immediately.

Theorem 3.3: Under Assumptions 2.1-2.3, if there exist positive-definite diagonal matrices $P$ and $R$ and constants $h>0$, $\alpha>0$, and $\beta>0$ such that the following conditions hold:

$$
\begin{aligned}
& {\left[\begin{array}{cccc}
-2 P \Gamma_{m}+\frac{2 h P}{d} & R \Delta & P & P \\
(R \Delta)^{T} & \Xi_{2} & 0 & 0 \\
P & 0 & -\alpha I & 0 \\
P & 0 & 0 & -\beta I
\end{array}\right]<0} \\
& \frac{2 h}{\bar{d}}>\frac{\alpha \delta_{M}^{2}\left(\left\|W_{*}\right\|+\left\|W^{*}\right\|\right)^{2}}{\lambda_{m}(P)}
\end{aligned}
$$

then the equilibrium point of system (36) is unique, and it is globally robustly exponentially stable, independent of time-varying delay, where $\Xi_{2}=\beta\left(\left\|W_{+}\right\|+\left\|W^{+}\right\|\right)^{2} I-2 R$.

Theorem 3.4: Under Assumptions 2.1-2.3, if there exist positive-definite diagonal matrices $P$ and $R$ and constants $h>1 / 2$, $\alpha>0$, and $\beta>0$ such that the following LMIs hold:

$$
\begin{aligned}
& {\left[\begin{array}{cccc}
\Xi_{3} & R \Delta & P & P \\
(R \Delta)^{T} & \Xi_{4} & 0 & 0 \\
P & 0 & -\beta I & 0 \\
P & 0 & 0 & \Xi_{5}
\end{array}\right]<0} \\
& P>\frac{\alpha \Delta \Delta \bar{d}}{\underline{d}}
\end{aligned}
$$

then the equilibrium point of system (36) is unique, and it is globally robustly exponentially stable, independent of time-varying delay, where $\Xi_{3}=-2 P \Gamma_{m}+2 h P / d$, $\Xi_{4}=\beta\left(\left\|W^{+}\right\|+\left\|W_{+}\right\|\right)^{2} I-2 R$, and $\Xi_{5}=-\alpha\left(\left\|W^{*}\right\|+\right.$ $\left.\left\|W_{*}\right\|\right)^{-2} I / \underline{d}$.

For the case of no uncertainties, we have the following results, respectively.

Corollary 3.4: Under Assumptions 2.1-2.3, if there exist positive-definite diagonal matrices $P, Q, R$, and $H$ and positive constant $\theta>0$ such that the following LMIs hold:

$$
\begin{aligned}
& {\left[\begin{array}{ccc}
\Phi_{3} & \Phi_{4} & P W_{1} \\
\Phi_{4}^{T} & Q W_{0}+W_{0}^{T} Q-2 R & Q W_{1} \\
W_{1}^{T} P & W_{1}^{T} Q & -H
\end{array}\right]<0} \\
& P \\
& \overline{\bar{d}}>\Delta H \Delta
\end{aligned}
$$

then the equilibrium point of system (36) without uncertainties is unique, and it is globally exponentially stable, independent of time-varying delay, where $\Phi_{3}=-2 P \Gamma+(P+Q \Delta) / \underline{d}+\theta I$ and $\Phi_{4}=P W_{0}-Q \Gamma+R \Delta$.

Corollary 3.5: Under Assumptions 2.1-2.3, if there exist positive-definite diagonal matrices $P, Q$, and $H$ and positive constant $\theta>0$ such that the following LMIs hold:

$$
\begin{aligned}
& {\left[\begin{array}{ccc}
\Phi_{5} & P W_{0} & P W_{1} \\
W_{0}^{T} P & \Phi_{6} & Q W_{1} \\
W_{1}^{T} P & W_{1}^{T} Q & -H
\end{array}\right]<0} \\
& \frac{P}{\bar{d}}>\Delta H \Delta
\end{aligned}
$$

then the equilibrium point of system (36) without uncertainties is unique, and it is globally exponentially stable, independent of time-varying delay, where $\Phi_{5}=-2 P \Gamma+(P+Q \Delta) / \underline{d}+\theta I$ and $\Phi_{6}=Q W_{0}+W_{0}^{T} Q-2 Q \Gamma \Delta^{-1}$.

Corollary 3.6: Under Assumptions 2.1-2.3, if there exist positive-definite diagonal matrices $P, Q$, and $R$ and a positive constant $h>0$ such that the following conditions hold:

$$
\begin{aligned}
& {\left[\begin{array}{ccc}
-2 P \Gamma+\frac{2 h P}{\underline{d}} & P W_{0}+R \Delta & P \\
\left(P W_{0}+R \Delta\right)^{T} & -2 R & 0 \\
P & 0 & -Q
\end{array}\right]<0} \\
& \frac{2 h}{\bar{d}}>\frac{\lambda_{M}(Q) \lambda_{M}\left(\Delta^{2}\right)\left\|W_{1}\right\|^{2}}{\lambda_{m}(P)}
\end{aligned}
$$


then the equilibrium point of system (36) without uncertainties is unique, and it is globally exponentially stable, independent of time-varying delay.

Corollary 3.7: Under Assumptions 2.1-2.3, if there exist positive-definite diagonal matrices $P, Q$, and $R$ and a positive constant $h>1 / 2$ such that the following LMIs hold:

$$
\begin{aligned}
& {\left[\begin{array}{ccc}
-2 P \Gamma+\frac{2 h P}{\underline{d}} & P W_{0}+R \Delta & P W_{1} \\
\left(P W_{0}+R \Delta\right)^{T} & -2 R & 0 \\
W_{1}^{T} P & 0 & -Q \underline{d}
\end{array}\right]<0} \\
& P>\frac{\Delta Q \Delta \bar{d}}{\underline{d}}
\end{aligned}
$$

then the equilibrium point of system (36) without uncertainties is unique, and it is globally exponentially stable, independent of time-varying delay.

Remark 3.2: When $d_{i}\left(u_{i}(t)\right) \equiv 1, a_{i}\left(u_{i}(t)\right)=c_{i} u_{i}(t)$ with $0<\underline{c}_{i} \leq c_{i} \leq \bar{c}_{i}, \bar{g}(\cdot)=\bar{f}(\cdot), \tau_{i j}(t)=\tau_{i j}$, or $\tau_{i j}(t)=\tau$, system (1) becomes

$$
\begin{aligned}
\frac{d u_{i}(t)}{d t}= & -c_{i} u_{i}(t)+\sum_{j=1}^{n} w_{0 i j} \bar{f}_{j}\left(u_{j}(t)\right) \\
& +\sum_{j=1}^{n} w_{1 i j} \bar{f}_{j}\left(u_{j}\left(t-\tau_{i j}\right)\right)+U_{i}
\end{aligned}
$$

or

$$
\begin{aligned}
\frac{d u_{i}(t)}{d t}= & -c_{i} u_{i}(t)+\sum_{j=1}^{n} w_{0 i j} \bar{f}_{j}\left(u_{j}(t)\right) \\
& +\sum_{j=1}^{n} w_{1 i j} \bar{f}_{j}\left(u_{j}(t-\tau)\right)+U_{i} .
\end{aligned}
$$

For models (54) and (55), robust stability problems were studied in [31]. For model (54), [31, Th. 3] requires

$$
\hat{\Omega}_{1}=2 \hat{r} I+\hat{S}-\|P\|_{2}\left(\|\hat{B}\|_{1}+\|\hat{B}\|_{\infty}\right) I>0
$$

and for model (55), [31, Th. 1] requires

$$
\hat{\Omega}_{2}=2 \hat{r} I+\hat{S}-\|P\|_{2}\left(\left\|W^{*}\right\|_{2}+\left\|W_{*}\right\|_{2}\right) I>0
$$

where $P=\operatorname{diag}\left(p_{i}, \ldots, p_{n}\right)$ is a positive diagonal matrix to be determined, $\hat{S}=\left(\hat{s}_{i j}\right)_{n \times n}, \hat{s}_{i i}=-2 p_{i} \bar{w}_{0 i i}$, $\hat{s}_{i j}=-\max \left(\left|p_{i} \bar{w}_{0 i j}+p_{j} \bar{w}_{0 j i}\right|,\left|p_{i} \underline{w}_{0 i j}+p_{j} \underline{w}_{0 j i}\right|\right)$ for $i \neq j$, $\hat{B}=\left(\hat{b}_{i j}^{*}\right), \hat{b}_{i j}^{*}=\max \left(\left|\bar{w}_{1 i j}\right|,\left|\underline{w}_{1 i j}\right|\right), \hat{r}=\min \left(p_{i} \underline{c}_{i} / \delta_{i}^{f}\right)$, and $\|\cdot\|_{e}$ denotes the suitable norm defined in [31], $e=1,2, \infty$. Comparing Corollary 3.1 of this paper with [31, Th. 3] and Theorem 3.2 of this paper with [31, Th. 1], the following differences can be observed. 1) Matrix norm method was used in [31], and the criteria in [31] are not easily verified because they involve the norm of an unknown matrix. In contrast, LMI technique is used in our paper, and the stability criteria can be easily checked using the interior point algorithms [4]. In general, matrix norm method and LMI technique are different approaches and usually lead to different sufficient conditions for the stability of neural network. 2) Some asymptotic stability criteria were derived in [31] for the case of constant delays, while some exponential stability criteria are derived in our paper. In summary, we have established a new set of robust stability criteria for the neural networks with delays.

\section{Robust Stability of COHEN-Grossberg NeURAL NETWORKS IN BIOLOGY}

In the original paper [12], Cohen-Grossberg neural network model was proposed as a kind of competitive-cooperation dynamical system, in which each state of neuron is always nonnegative for all time. It is clear that this subset of Cohen-Grossberg neural networks includes the famous Lotka-Volterra recurrent neural networks [29], [30].

When model (1) is applied to biological systems, the initial condition for (1) is of the following type:

$$
\begin{aligned}
u_{i}(t) & =\phi_{i}(t) \geq 0, \quad-\rho \leq t \leq 0 \\
u_{i}(0) & =\phi_{i}(0)>0
\end{aligned}
$$

where each $\phi_{i}(\cdot), i=1, \ldots, n$, is a continuous function defined on $[-\rho, 0]$.

In applications, the activation functions of model (1) may not be bounded, for example, the Lotka-Volterra model [40], [41]. Therefore, in this section, we need the following assumptions and lemmas.

Assumption 4.1: Activation functions $\bar{g}_{i}(\cdot)$ and $\bar{f}_{i}(\cdot)$ satisfy conditions (2) and (3), $a_{i}(\cdot)$ satisfies (4), respectively, and $\bar{g}_{i}(0)=0, \bar{f}_{i}(0)=0$, and $a_{i}(0)=0, i=1, \ldots, n$.

Assumption 4.2: The amplification function $d_{i}(\varrho)>0$ for all $\varrho>0$ and $d_{i}(0)=0$, and for any $\epsilon>0, \int_{0}^{\epsilon}\left(d \varrho / d_{i}(\varrho)\right)=+\infty$ and $\int_{\epsilon_{0}}^{\epsilon_{1}}\left(d \varrho / d_{i}(\varrho)\right)<+\infty$ for all $i=1, \ldots, n$, where $\epsilon_{0}$ and $\epsilon_{1}$ are bounded positive constants.

Lemma 4.1: Assume that $d_{i}(\varrho)$ satisfies Assumption 4.2, then the solution of the system (1) with the initial condition (56) is a positive function.

Proof: In a similar routine to the proof of [29, Lemma 4] or [30, Lemma 1], Lemma 4.1 can be proved. The details are omitted.

If Assumption 4.2 holds, then the nonnegative equilibrium point of system (1) is a solution of the following equation:

$$
u_{i}\left[F_{i}(u)+U_{i}\right]=0, \quad i=1, \ldots, n
$$

where

$$
F_{i}(u)=a_{i}\left(u_{i}\right)-\sum_{j=1}^{n} w_{0 i j} \bar{g}_{j}\left(u_{j}\right)-\sum_{j=1}^{n} w_{1 i j} \bar{f}_{j}\left(u_{j}\right) .
$$

Though (57) might possess multiple solutions, in a similar routine to the proof of Proposition 1 in [30], we can show that if $u_{i}^{*}$ is an asymptotical stable nonnegative equilibrium point of system (1), then it must be a solution of the following problem:

$$
u_{i}^{*} \geq 0 \quad F_{i}\left(u^{*}\right)+U_{i} \geq 0 \quad u_{i}^{*}\left(F_{i}\left(u^{*}\right)+U_{i}\right)=0
$$

where $i=1, \ldots, n$.

Lemma 4.2 (See [30]): Equation (58) has a unique solution for every $U_{i}$ if and only if $\bar{F}(u)$ is norm coercive, i.e.,

$$
\lim _{\|u\| \rightarrow \infty}\|\bar{F}(u)\|=\infty
$$


and locally univalent, where $\bar{F}(u)=F\left(u^{+}\right)+u^{-}$, $F(u)=\left(F_{1}(u), \ldots, F_{n}(u)\right)^{T}, u^{+}=\left(u_{1}^{+}, u_{2}^{+}, \ldots, u_{n}^{+}\right)^{T}$, $u^{-}=\left(u_{1}^{-}, u_{2}^{-}, \ldots, u_{n}^{-}\right)^{T}, u_{i}^{+}=u_{i}$ if $u_{i} \geq 0$ and $u_{i}^{+}=0$ if $u_{i}<0, u_{i}^{-}=u_{i}$ if $u_{i} \leq 0$, and $u_{i}^{-}=0$ if $u_{i}>0, i=1, \ldots, n$.

Definition 4.1: $u^{*}$ is said to be a nonnegative equilibrium point of system (1) if $u^{*}$ is a solution of (58). Moreover, if $u_{i}^{*}>$ 0 for all $i=1, \ldots, n$, then $u^{*}$ is said to be a positive equilibrium point of system (1). In this case, $u^{*}$ must satisfy

$$
A\left(u^{*}\right)-W_{0} \bar{g}\left(u^{*}\right)-W_{1} \bar{f}\left(u^{*}\right)+U=0
$$

where $A\left(u^{*}\right)=\left(a_{1}\left(u_{1}^{*}\right), \ldots, a_{n}\left(u_{n}^{*}\right)\right)^{T}, \bar{g}\left(u^{*}\right)=$ $\left(\bar{g}_{1}\left(u_{1}^{*}\right), \ldots, \bar{g}_{n}\left(u_{n}^{*}\right)\right)^{T}, \bar{f}\left(u^{*}\right)=\left(\bar{f}_{1}\left(u_{1}^{*}\right), \ldots, \bar{f}_{n}\left(u_{n}^{*}\right)\right)^{T}$, and $U=\left(U_{1}, \ldots, U_{n}\right)^{T}$.

Definition 4.2 (See [6] and [29]): Let $R$ be an $n \times n$ matrix, and $\left.R=\left(R_{i j}\right) \mid R_{i j} \leq 0, i \neq j\right)$, then $R$ is a nonsingular $M$-matrix if one of the following conditions holds: 1) All the leading principle minors of $R$ are positive. 2) $R$ has all positive diagonal elements and there exists a positive diagonal matrix $\Lambda=\operatorname{diag}\left(\lambda_{1}, \ldots, \lambda_{n}\right)$ such that $R \Lambda$ is strictly diagonally dominant. 3) $R$ is quasi-dominant positive diagonal, i.e., there exist positive numbers $z_{j}$ such that $\sum_{j=1}^{n} z_{j} R_{i j}>0$ or $\sum_{j=1}^{n} R_{j i} z_{j}>0$. That is, $R Z>0$ or $Z^{T} \stackrel{R}{R}>0$, where $Z=\left(z_{1}, z_{2}, \ldots, z_{n}\right)^{T}$.

For any nonsingular $M$-matrix $R$, let $\Omega_{M}(R)=$ $\left\{Z \in R_{+}^{n} \mid R Z>0\right\}$, where $R_{+}^{n}=\left\{x \in R^{n} \mid x>0\right\}$. Clearly, $\Omega_{M}(R)$ is nonempty and $k_{1} Z_{1}+k_{2} Z_{2} \in \Omega_{M}(R)$ for any scalars $k_{1}>0$ and $k_{2}>0$ and vectors $Z_{1}, Z_{2} \in \Omega_{M}(R)$.

The following theorem gives a condition for the existence and uniqueness of the equilibrium point of (1).

Theorem 4.1: Suppose Assumptions 4.1-4.2 hold. Then, system (1) with initial condition (56) has a unique positive equilibrium point if there exist positive constants $L_{1}, L_{2}, \ldots, L_{n}$ such that the following inequality holds:

$$
\begin{aligned}
L_{i} \Gamma_{m} & -\frac{1}{2} \sum_{j=1}^{n}\left(L_{i} w_{0 i j}^{*} \delta_{j}^{g}+L_{j} w_{0 j i}^{*} \delta_{i}^{g}\right) \\
& -\frac{1}{2} \sum_{j=1}^{n}\left(L_{i} w_{1 i j}^{*} \delta_{j}^{f}+L_{j} w_{1 j i}^{*} \delta_{i}^{f}\right)>0, \quad i=1, \ldots, n .
\end{aligned}
$$

where $w_{0 i j}^{*}=\max \left(\left|\underline{w}_{0 i j}\right|,\left|\bar{w}_{0 i j}\right|\right)$ and $w_{1 i j}^{*}=$ $\max \left(\left|\underline{w}_{1 i j}\right|,\left|\bar{w}_{1 i j}\right|\right), i, j=1, \ldots, n$.

Proof: According to Lemma 4.2, we only need to prove that $\bar{F}(u)$ is norm coercive and locally univalent. First, we prove $\bar{F}(u)$ is locally univalent. For any $u=\left(u_{1}, \ldots, u_{n}\right) \in \Re^{n}$, without loss of generality, by some rearrangement of $u_{i}$, we can assume $u_{i}>0$ if $i=1, \ldots, p, u_{i}<0$ if $i=p+1, \ldots, m$, and $u_{i}=0$ if $i=m+1, \ldots, n$, for some integers $p \leq m \leq n$. Moreover, if $y \in \Re^{n}$ is sufficiently close to $u \in \Re^{n}$, without loss of generality, we can also assume $y_{i}>0$ if $i=1, \ldots, p$, $y_{i}<0$ if $i=p+1, \ldots, m, y_{i}>0$ if $i=m+1, \ldots, m_{1}, y_{i}<0$ if $i=m_{1}+1, \ldots, m_{2}$, and $y_{i}=0$ if $i=m_{2}+1, \ldots, n$, for some integers $m \leq m_{1} \leq m_{2} \leq n$. It can be seen that

$$
\left(u_{i}^{+}-y_{i}^{+}\right)\left(u_{i}^{-}-y_{i}^{-}\right)=0, \quad i=1, \ldots, n .
$$

If $\bar{F}_{i}(u)-\bar{F}_{i}(y)=0$, then we have

$$
\begin{aligned}
\Gamma_{m}\left|u_{i}^{+}-y_{i}^{+}\right| \leq & \left|a_{i}\left(u_{i}^{+}\right)-a_{i}\left(y_{i}^{+}\right)\right| \\
\leq & \sum_{j=1}^{n}\left(w_{0 i j}^{*} \delta_{j}^{g}\left|u_{j}^{+}-y_{j}^{+}\right|+w_{1 i j}^{*} \delta_{j}^{f}\left|u_{j}^{+}-y_{j}^{+}\right|\right) \\
& +\left|u_{i}^{-}-y_{i}^{-}\right| .
\end{aligned}
$$

Multiplying $L_{i}\left|u_{i}^{+}-y_{i}^{+}\right|$on both sides of (61), it yields

$$
\begin{aligned}
& \Gamma_{m} L_{i}\left|u_{i}^{+}-y_{i}^{+}\right|^{2} \\
& \leq \sum_{j=1}^{n} w_{0 i j}^{*} \delta_{j}^{g} L_{i}\left|u_{i}^{+}-y_{i}^{+}\right|\left|u_{j}^{+}-y_{j}^{+}\right| \\
& \quad+\sum_{j=1}^{n} w_{1 i j}^{*} \delta_{j}^{f} L_{i}\left|u_{i}^{+}-y_{i}^{+}\right|\left|u_{j}^{+}-y_{j}^{+}\right|, \\
& \leq \frac{\sum_{j=1}^{n} w_{0 i j}^{*} \delta_{j}^{g} L_{i}\left(\left|u_{i}^{+}-y_{i}^{+}\right|^{2}+\left|u_{j}^{+}-y_{j}^{+}\right|^{2}\right)}{2} \\
& \quad+\frac{\sum_{j=1}^{n} w_{1 i j}^{*} \delta_{j}^{f} L_{i}\left(\left|u_{i}^{+}-y_{i}^{+}\right|^{2}+\left|u_{j}^{+}-y_{j}^{+}\right|^{2}\right)}{2}
\end{aligned}
$$

where we have used condition (60). It follows from (62) that the following condition holds:

$$
\begin{gathered}
\left\{\sum_{i=1}^{n} \Gamma_{m} L_{i}-\frac{1}{2} \sum_{i=1}^{n} \sum_{j=1}^{n}\left[\left(L_{i} w_{0 i j}^{*} \delta_{j}^{g}+L_{j} w_{0 j i}^{*} \delta_{i}^{g}\right)\right]\right. \\
\left.-\frac{1}{2} \sum_{i=1}^{n} \sum_{j=1}^{n}\left[\left(L_{i} w_{1 i j}^{*} \delta_{j}^{f}+L_{j} w_{1 j i}^{*} \delta_{i}^{f}\right)\right]\right\}\left|u_{i}^{+}-y_{i}^{+}\right|^{2} \\
=\sum_{i=1}^{n}\left[\Gamma_{m} L_{i}-\frac{1}{2} \sum_{j=1}^{n}\left(L_{i} w_{0 i j}^{*} \delta_{j}^{g}+L_{j} w_{0 j i}^{*} \delta_{i}^{g}\right)\right. \\
\left.\quad-\frac{1}{2} \sum_{j=1}^{n}\left(L_{i} w_{1 i j}^{*} \delta_{j}^{f}+L_{j} w_{1 j i}^{*} \delta_{i}^{f}\right)\right]\left|u_{i}^{+}-y_{i}^{+}\right|^{2} \\
\leq 0 .
\end{gathered}
$$

By (59), it comes $\left|u_{i}^{+}-y_{i}^{+}\right|^{2} \leq 0$, which means $u^{+}=y^{+}$. In a similar manner to the proof of Theorem 2 in [30], we can also show $u^{-}=y^{-}$. This implies that $u=y$. Therefore, $\bar{F}(u)$ is locally univalent.

Next, we will prove that $\bar{F}(u)$ is norm coercive. Because

$$
\begin{aligned}
\bar{F}_{i}(u)= & a_{i}\left(u_{i}^{+}\right)-\sum_{j=1}^{n} w_{0 i j} \bar{g}_{j}\left(u_{j}^{+}\right)-\sum_{j=1}^{n} w_{1 i j} \bar{f}_{j}\left(u_{j}^{+}\right)+u_{i}^{-} \\
= & \left(a_{i}\left(u_{i}^{+}\right)-a_{i}(0)\right)-\sum_{j=1}^{n} w_{0 i j}\left(\bar{g}_{j}\left(u_{j}^{+}\right)-\bar{g}_{j}(0)\right) \\
& -\sum_{j=1}^{n} w_{1 i j}\left(\bar{f}_{j}\left(u_{j}^{+}\right)-\bar{f}_{j}(0)\right)+u_{i}^{-}
\end{aligned}
$$

multiplying $u_{i}^{+} L_{i}$ on both sides of (64), we have

$$
\begin{aligned}
& \sum_{i=1}^{n}\left|u_{i}^{+} L_{i} \bar{F}_{i}(u)\right| \\
& \quad=\sum_{i=1}^{n} \mid u_{i}^{+} L_{i}\left(a_{i}\left(u_{i}^{+}\right)-a_{i}(0)\right)
\end{aligned}
$$




$$
\begin{aligned}
& -u_{i}^{+} L_{i} \sum_{j=1}^{n} w_{0 i j}\left(\bar{g}_{j}\left(u_{j}^{+}\right)-\bar{g}_{j}(0)\right) \\
& -u_{i}^{+} L_{i} \sum_{j=1}^{n} w_{1 i j}\left(\bar{f}_{j}\left(u_{j}^{+}\right)-\bar{f}_{j}(0)\right) \mid \\
& \geq \sum_{i=1}^{n}\left[L_{i} \Gamma_{m}\left|u_{i}^{+}\right|^{2}-L_{i}\left|u_{i}^{+}\right| \sum_{j=1}^{n} w_{0 i j}^{*} \delta_{j}^{g}\left|u_{j}^{+}\right|\right. \\
& \left.-L_{i}\left|u_{i}^{+}\right| \sum_{j=1}^{n} w_{1 i j}^{*} \delta_{j}^{f}\left|u_{j}^{+}\right|\right] \\
& \geq \sum_{i=1}^{n}\left[L_{i} \Gamma_{m}\left|u_{i}^{+}\right|^{2}-\frac{L_{i} \sum_{j=1}^{n} w_{0 i j}^{*} \delta_{j}^{g}\left(\left|u_{i}^{+}\right|^{2}+\left|u_{j}^{+}\right|^{2}\right)}{2}\right. \\
& \left.-\frac{L_{i} \sum_{j=1}^{n} w_{1 i j}^{*} \delta_{j}^{f}\left(\left|u_{i}^{+}\right|^{2}+\left|u_{j}^{+}\right|^{2}\right)}{2}\right] \\
& =\sum_{i=1}^{n}\left[L_{i} \Gamma_{m}-\frac{1}{2} \sum_{j=1}^{n}\left(L_{i} w_{0 i j}^{*} \delta_{j}^{g}+L_{j} w_{0 j i}^{*} \delta_{i}^{g}\right)\right. \\
& \left.-\frac{1}{2} \sum_{j=1}^{n}\left(L_{i} w_{1 i j}^{*} \delta_{j}^{f}+L_{j} w_{1 j i}^{*} \delta_{i}^{f}\right)\right]\left|u_{i}^{+}\right|^{2} .
\end{aligned}
$$

Considering (59), from (65), we have

$$
\left\|u^{+}\right\|\|L\|\|\bar{F}(u)\| \geq\left(u^{+}\right)^{T} L \bar{F}(u) \geq r_{0}\left\|u^{+}\right\|^{2}
$$

where

$$
\begin{aligned}
0<r_{0}=\min _{1 \leq i \leq n}\left(L_{i} \Gamma_{m}-\frac{1}{2} \sum_{j=1}^{n}\left(L_{i} w_{0 i j}^{*} \delta_{j}^{g}+L_{j} w_{0 j i}^{*} \delta_{i}^{g}\right)\right. \\
\left.-\frac{1}{2} \sum_{j=1}^{n}\left(L_{i} w_{1 i j}^{*} \delta_{j}^{f}+L_{j} w_{1 j i}^{*} \delta_{i}^{f}\right)\right) .
\end{aligned}
$$

Therefore, $\|\bar{F}(u)\| \geq r_{0}\|u\| /\|L\| \rightarrow \infty$ as $\|u\| \rightarrow \infty$, which implies that $\bar{F}(u)$ is norm coercive. In combination with Lemma 4.2, (59) ensures the existence and uniqueness of the positive equilibrium point of system (1).

Let $u^{*}=\left[u_{1}^{*}, \ldots, u_{n}^{*}\right]^{T}$ be an equilibrium point of system (1) and $x_{i}(t)=u_{i}(t)-u_{i}^{*}$, then model (1) is transformed into the following form:

$$
\begin{aligned}
\dot{x}_{i}(t)=-D_{i}\left(x_{i}(t)\right)[ & A_{i}\left(x_{i}(t)\right)-\sum_{j=1}^{n} w_{0 i j} g_{j}\left(x_{j}(t)\right) \\
& \left.-\sum_{j=1}^{n} w_{1 i j} f_{j}\left(x_{j}\left(t-\tau_{i j}(t)\right)\right)+J_{i}\right]
\end{aligned}
$$

where $J_{i}=J_{i}^{s}$ if $u_{i}^{*}=0, J_{i}=0$ if $u_{i}^{*}>0, J_{i}^{s}=a_{i}\left(u_{i}^{*}\right)-$ $\sum_{j=1}^{n} w_{0 i j} \bar{g}_{j}\left(u_{j}^{*}\right)-\sum_{j=1}^{n} w_{1 i j} \bar{f}_{j}\left(u_{j}^{*}\right)+U_{i}$, initial condition satisfies (56), and others are defined in (7).

According to (58), $J_{i} \geq 0$ holds for all $i=1, \ldots, n$. This implies that $x_{i}(t) J_{i} \geq 0$ and $f_{i}\left(x_{i}(t)\right) J_{i} \geq 0$ hold for all $i=$ $1, \ldots, n$ and $t \geq 0$.

Lemma 4.1 shows that the concerned Cohen-Grossberg neural network (1) possesses the property that all trajectories with initial condition (56) stay in the positive region. Therefore, the remainder of this section is only concerned with the robust stability of the positive equilibrium point of system (1) with initial condition (56).
Theorem 4.2: Suppose Assumptions 4.1-4.2 hold. Then, the positive equilibrium point of system (1) with initial condition (56) is globally robustly stable if the following matrix:

$$
\bar{\Psi}=\left(\bar{\psi}_{i j}\right)_{n \times n}=\Gamma-W_{0}^{*} \Delta_{g}-W_{1}^{*} \Delta_{f}
$$

is a $M$-matrix, where the notations are the same as those in Theorem 4.1.

Proof: This theorem can be proved following a similar routine to the proof of Theorems 2 and 4 in [29]. Due to the limitation of space, details are omitted.

\section{SimUlation EXAMPLES}

In this section, we will give two examples to show the effectiveness of the present results.

1) Example 5.1: Consider the interval Cohen-Grossberg neural networks (1), where

$$
\begin{aligned}
& \underline{W}_{0}=\left[\begin{array}{ccc}
-0.06 & 0.3 & 0.03 \\
0 & -0.03 & -0.06 \\
0.03 & -0.03 & 0
\end{array}\right] \\
& \bar{W}_{0}=\left[\begin{array}{ccc}
-0.015 & 0.333 & 0.15 \\
0.003 & 0.006 & -0.03 \\
0.09 & -0.006 & 0.03
\end{array}\right] \\
& \underline{W}_{1}=\left[\begin{array}{ccc}
-0.045 & -0.03 & 0.036 \\
0 & -0.3 & -0.03 \\
0.12 & 0.3 & 0.051
\end{array}\right] \\
& \bar{W}_{1}=\left[\begin{array}{ccc}
-0.03 & 0.0048 & 0.09 \\
0.015 & 0.048 & 0 \\
0.3 & 0.45 & 0.057
\end{array}\right]
\end{aligned}
$$

$\delta_{i}^{g}=\delta_{i}^{f}=1.2, \bar{d}=1.4, \underline{d}=1, \Gamma_{m}=2$, and $\tau_{i j}(t)>0$ is any bounded and unknown time-varying delays $i, j=1,2,3$.

Because $\delta_{i}^{g}>1$ and $\tau_{i j}(t)>0$ are any unknown time-varying delays, the results in [11] are not suitable for this example. Applying Theorem 3.1 of this paper, we have $P=14.6866 I, Q=0.2813 I, M=0.2173 I$, $R_{1}=R_{2}=R_{3}=1.6226 I, S_{1}=S_{2}=S_{3}=0.2547 I$, $\theta=0.0480, \alpha=28.1377, \beta=0.1895, \varepsilon=0.7006$, $\beta_{1}=0.7771, \beta_{2}=2.1572, \beta_{3}=3.9152, \gamma_{1}=0.0512$, $\gamma_{2}=0.0775, \gamma_{3}=0.1169, \varepsilon_{1}=0.0458, \varepsilon_{2}=0.0504$, and $\varepsilon_{3}=0.0599$. Therefore, the system in this example is globally robustly exponentially stable.

2) Example 5.2: Consider the following two Cohen-Grossberg neural networks with two neurons:

$$
\begin{aligned}
& \dot{y}_{1}(t) \\
& =y_{1}(t)\left[-6 y_{1}(t)+w_{011} g_{1}\left(y_{1}(t)\right)+w_{012} g_{2}\left(y_{2}(t)\right)\right. \\
& \left.+w_{111} g_{1}\left(y_{1}(t-2)\right)+w_{112} g_{2}\left(y_{2}(t-3)\right)+1\right] \\
& =y_{2}(t)\left[-6 y_{2}(t)+w_{021} g_{1}\left(y_{1}(t)\right)+w_{022} g_{2}\left(y_{2}(t)\right)\right. \\
& \left.+w_{121} g_{1}\left(y_{1}(t-2)\right)+w_{122} g_{2}\left(y_{2}(t-3)\right)+1\right]
\end{aligned}
$$




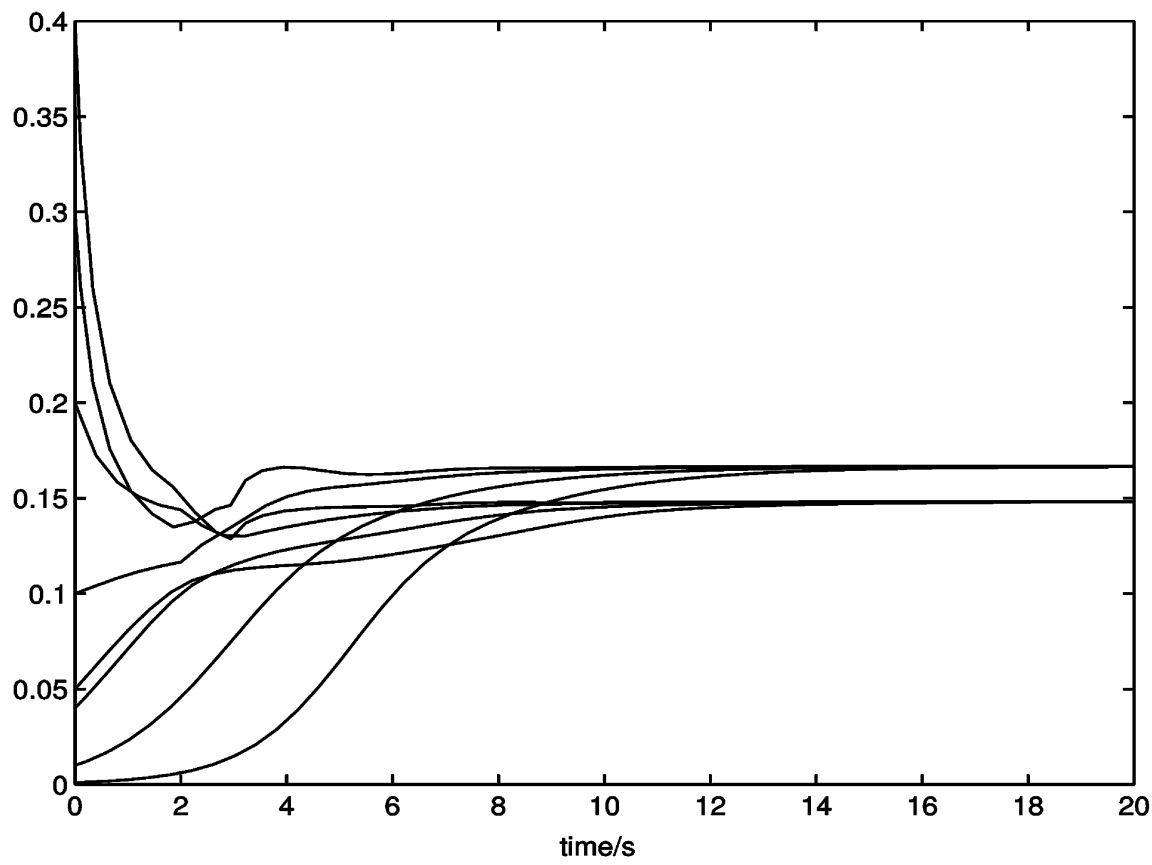

Fig. 1. State response curves of Example 5.2 with different initial values.

where $d_{i}\left(y_{i}\right)=y_{i}, g_{i}\left(y_{i}\right)=0.5\left[y_{i}+\tanh \left(y_{i}\right)\right], i=1,2$, $W_{0}=\left[\begin{array}{cc}-2 & 1 \\ -1 & -1\end{array}\right]$, and $W_{1}=\left[\begin{array}{cc}-1 & 1 \\ 1 & 1\end{array}\right]$.

Applying Theorem 4.2 of this paper, it yields

$$
\Gamma-\left|W_{0}\right| \Delta_{g}-\left|W_{1}\right| \Delta_{g}=\left[\begin{array}{cc}
3 & -2 \\
-2 & 4
\end{array}\right]
$$

and its eigenvalue is 1.4384 and 5.5616, respectively. Obviously, it is an $M$-matrix, and the equilibrium point of Cohen-Grossberg (69) is globally asymptotically stable.

The solutions of system (69) are $(0,0)^{T},(0,0.1667)^{T}$, $(0.1112,0)^{T}$, and $(0.1482,0.1667)^{T}$. Among them, $(0.1482,0.1667)^{T}$ is the unique positive equilibrium point.

When initial condition are $(0.04,0.01)^{T},(0.05,0.001)^{T}$, $(0.2,0.1)^{T}$, and $(0.4,0.3)^{T}$ for $s \in[-3,0]$, the response curves of system (69) are depicted in Fig. 1.

\section{CONCLUSION}

New global robust stability criteria are established for the interval Cohen-Grossberg neural networks with time-varying delays. All these criteria are independent of time-varying delays and have no restrictions on the value of the derivative of time-varying delays. Furthermore, the present results improve upon some existing results, and are less conservative than the existing results, as demonstrated using two numerical examples.

\section{REFERENCES}

[1] S. Arik, "An analysis of exponential stability of delayed neural networks with time varying delays," Neural Netw., vol. 17, pp. 1027-1031, 2004.

[2] S. Arik, "Global robust stability analysis of neural networks with discrete time delays," Chaos Solitons Fractals, vol. 26, pp. 1407-1414, 2005.

[3] S. Arik and Z. Orman, "Global stability analysis of Cohen-Grossberg neural networks with time varying delays," Phys. Lett. A, vol. 341, pp. 410-421, 2005.
[4] S. Boyd, L. E. Ghaoui, E. Feron, and V. Balakrishnan, Linear Matrix Inequality in System and Control Theory. Philadelphia, PA: SIAM, 1994.

[5] J. Cao, D. Huang, and Y. Qu, "Global robust stability of delayed recurrent neural networks," Chaos Solitons Fractals, vol. 23, pp. 221-229, 2005.

[6] J. Cao and J. Wang, "Global asymptotic stability of a general class of recurrent neural networks with time-varying delays," IEEE Trans. Circuits Syst. I, Fundam.l Theory Appl., vol. 50, no. 1, pp. 34-44, Jan. 2003.

[7] J. Cao and J. Wang, "Absolute exponential stability of recurrent neural networks with Lipschitz continuous activation functions and time delays," Neural Netw., vol. 17, pp. 379-390, 2004.

[8] J. Cao and J. Wang, "Global asymptotic and robust stability of recurrent neural networks with time delays," IEEE Trans. Circuits Syst. I, Reg. Papers, vol. 52, no. 2, pp. 417-426, Feb. 2005.

[9] Y. Chen, "Global asymptotic stability of delayed Cohen-Grossberg neural networks," IEEE Trans. Circuits Syst. I, Reg. Papers, vol. 53, no. 2, pp. 351-357, Feb. 2006.

[10] T. Chen and L. Rong, "Delay-independent stability analysis of Cohen-Grossberg neural networks," Phys. Lett. A, vol. 317, pp. 436-449, 2003.

[11] T. Chen and L. Rong, "Robust global exponential stability of Cohen-Grossberg neural networks with time delays," IEEE Trans. Neural Netw., vol. 15, no. 1, pp. 203-206, Jan. 2004.

[12] M. A. Cohen and S. Grossberg, "Stability and global pattern formulation and memory storage by competitive neural networks," IEEE Trans. Syst. Man Cybern., vol. SMC-13, no. 5, pp. 815-826, Sep.-Oct. 1983.

[13] T. Ensari and S. Arik, "Global stability of a class of neural networks with time varying delay," IEEE Trans. Circuits Syst. II, Exp. Briefs, vol. 52, no. 3, pp. 126-130, Mar. 2005.

[14] M. Forti, P. Nistri, and M. Quincampoix, "Generalized neural network for nonsmooth nonlinear programming problems," IEEE Trans. Circuits Syst. I, Reg. Papers, vol. 51, no. 9, pp. 1741-1754, Sep. 2004.

[15] M. Forti, P. Nistri, and D. Papini, "Global exponential stability and global convergence in finite time of delayed neural networks with infinite gain," IEEE Trans. Neural Netw., vol. 16, no. 6, pp. 1449-1463, Nov. 2005.

[16] S. Guo and L. Huang, "Stability analysis of Cohen-Grossberg neural networks," IEEE Trans. Neural Netw., vol. 17, no. 1, pp. 106-117, Jan. 2006.

[17] Y. He, M. Wu, and J. H. She, "An improved global asymptotic stability criterion for delayed cellular neural networks," IEEE Trans. Neural Netw., vol. 17, no. 1, pp. 1250-1252, Jan. 2006. 
[18] J. J. Hopfield, "Neurons with graded response have collective computational properties like those of two-state neurons," Proc. Nat. Acad. Sci., vol. 81, no. 10, pp. 3088-3092, 1984.

[19] C. C. Hwang, C. J. Chang, and T. L. Liao, "Globally exponential stability of generalized Cohen-Grossberg neural networks with delays," Phys. Lett. A, vol. 319, pp. 157-166, 2003.

[20] C. Ji, H. Zhang, and Y. Wei, "LMI approach for global robust stability of Cohen-Grossberg neural networks with multiple delays," Neurocomputing, vol. 71, no. 4, pp. 475-485, 2008.

[21] C. Li, X. Liao, and Y. Chen, "On robust stability of BAM neural networks with constant delays," in Lectures Notes in Computer Science, F. Yin, J. Wang, and C. Guo, Eds. Berlin, Germany: Springer-Verlag, 2004, vol. 3173, pp. 102-107.

[22] T. Li, L. Guo, C. Sun, and C. Lin, "Further results on delay-dependent stability criteria of neural networks with time-varying delays," IEEE Trans. Neural Netw., vol. 19, no. 4, pp. 726-730, Apr. 2008.

[23] X. Liao, G. Chen, and E. N. Sanchez, "LMI-based approach for asymptotically stability analysis of delayed neural networks," IEEE Trans. Circuits Syst. I, Fundam. Theory Appl., vol. 49, no. 7, pp. 1033-1039, Jul. 2002.

[24] X. Liao, G. Chen, and E. N. Sanchez, "Delay-dependent exponential stability analysis of delayed neural networks: An LMI approach," Neural Netw., vol. 15, pp. 855-866, 2002.

[25] X. Liao, K. Wong, Z. Wu, and G. Chen, "Novel robust stability criteria for interval delayed Hopfield neural networks," IEEE Trans. Circuits Syst. I, Fundam. Theory Appl., vol. 48, no. 11, pp. 1355-1358, Nov. 2001.

[26] X. Liao and J. Yu, "Robust stability for interval Hopfield neural networks with time delay," IEEE Trans. Neural Netw., vol. 9, no. 5, pp. 1042-1046, Sep. 1998.

[27] H. Lu, "Global exponential stability analysis of Cohen-Grossberg neural networks," IEEE Trans. Circuits Syst. II, Exp. Briefs, vol. 52, no. 8 , pp. 476-479, Aug. 2005.

[28] H. Lu, R. Shen, and F. Chung, "Global exponential convergence of Cohen-Grossberg neural networks with time delays," IEEE Trans. Neural Netw., vol. 16, no. 6, pp. 1694-1696, Nov. 2005.

[29] K. Lu, D. Xu, and Z. Yang, "Global attraction and stability for Cohen-Grossberg neural networks with delays," Neural Netw., vol. 19, pp. 1538-1549, 2006.

[30] W. Lu and T. Chen, " $\Re_{+}^{n}$ global stability of Cohen-Grossberg neural network system with nonnegative equilibria," Neural Netw., vol. 20, pp. 714-722, 2007.

[31] N. Ozcan and S. Arik, "Global robust stability analysis of neural networks with multiple time delays," IEEE Trans. Circuits Syst. I, Reg. Papers, vol. 53, no. 1, pp. 166-176, 2006.

[32] L. Rong, "LMI-based criteria for robust stability of Cohen-Grossberg neural networks with delay," Phys. Lett. A, vol. 339, pp. 63-73, 2005.

[33] H. Shao, "Delay-dependent approaches to globally exponential stability for recurrent neural networks," IEEE Trans. Circuits Syst. II, Exp. Briefs, vol. 55, no. 6, pp. 591-595, Jun. 2008.

[34] V. Singh, "Global robust stability of delayed neural networks: An LMI approach," IEEE Trans. Circuits Syst. II, Exp. Briefs, vol. 52, no. 1, pp. 33-36, Jan. 2005.

[35] Q. Song and J. Cao, "Global exponential robust stability of Cohen-Grossberg neural network with time-varying delays and reaction-diffusion terms," J. Franklin Inst., vol. 343, pp. 705-719, 2006.

[36] L. Wang and X. Zou, "Exponential stability of Cohen-Grossberg neural networks," Neural Netw., vol. 15, pp. 415-422, 2002.

[37] L. Wang and X. Zou, "Harmless delays in Cohen-Grossberg neural networks," Physica D, vol. 170, pp. 162-173, 2002.

[38] Z. Wang, H. Zhang, and W. Yu, "Robust exponential stability analysis of neural networks with multiple time delays," Neurocomputing, vol. 70, no. 13, pp. 2534-2543, 2007.

[39] S. Xu, Y. Chu, and J. Lu, "New results on global exponential stability of recurrent neural networks with time varying delays," Phys. Lett. A, vol. 352, pp. 371-379, 2006.

[40] Z. Yi and K. K. Tan, "Dynamic stability conditions for Lotka-Volterra recurrent neural networks with delays," Phys. Rev. E, Stat. Phys. Plasmas Fluids Relat. Interdiscip. Top., vol. 66, pp. 011-910, 2002.

[41] Z. Yi and K. K. Tan, "Global convergence of Lotka-Volterra recurrent neural networks with delays," IEEE Trans. Circuits Syst. I, Reg. Papers, vol. 52, no. 11, pp. 2482-2489, Nov. 2005.

[42] K. Yuan and J. Cao, "An analysis of global asymptotic stability of delayed Cohen-Grossberg neural networks via nonsmooth analysis," IEEE Trans. Circuits Syst. I, Reg. Papers, vol. 52, no. 9, pp. 1854-1861, Sep. 2005.

[43] E. Yucel and S. Arik, "New exponential stability results for delayed neural networks with time varying delays," Physica D, vol. 191, pp. 314-322, 2004.

[44] H. Zhang, Z. Wang, and D. Liu, "Exponential stability analysis of neural networks with multiple time delays," in Lectures Notes in Computer Science, J. Wang, X. Liao, and Z. Yi, Eds. Berlin, Germany: Springer-Verlag, 2005, vol. 3496, pp. 142-148.

[45] H. Zhang and Z. Wang, "Global asymptotic stability of delayed cellular neural networks," IEEE Trans. Neural Netw., vol. 18, no. 3, pp. 947-950, May 2007.

[46] H. Zhang, Z. Wang, and D. Liu, "Global asymptotic stability of recurrent neural networks with multiple time-varying delays," IEEE Trans. Neural Netw., vol. 19, no. 5, pp. 855-873, May 2008.

[47] J. Zhang, Y. Suda, and H. Komine, "Global exponential stability of Cohen-Grossberg neural networks with variable delays," Phys. Lett. A, vol. 338, pp. 44-50, 2005.

[48] D. Zhou and J. Cao, "Globally exponential stability conditions for cellular neural networks with time-varying delays," Appl. Math. Comput., vol. 131, pp. 487-496, 2002.

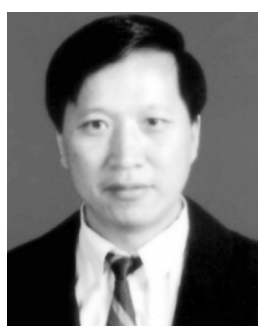

Huaguang Zhang (SM'04) received the B.S. and M.S. degrees in control engineering from Northeastern Electric Power University, Jilin, China, in 1982 and 1985, respectively, and the Ph.D. degree in thermal power engineering and automation from Southeast University, Nanjing, China, in 1991.

He joined the Department of Automatic Control, Northeastern University, Shenyang, China, in 1992, as a Postdoctoral Fellow. Since 1994, he has been a Professor and the Head of the Electric Automation Institute, Northeastern University. He has authored and coauthored over 200 journal and conference papers and four monographs. $\mathrm{He}$ holds nine patents. His main research interests are neural-network-based control, fuzzy control, chaos control, nonlinear control, signal processing, and their industrial applications.

Dr. Zhang is an Associate Editor of Automatica and Neurocomputing. He was Program Chair for the 2007 International Symposium on Neural Networks (Nanjing, China). He is currently an Associate Editor of the IEEE TRANSACtions ON SYSTEMS, Man, AND CYBERnETICS-PART B: Cyberneticsand the IEEE TRANSACTIONS ON FuZZy Systems. He is Program Chair for the 2009 IEEE International Conference on Automation and Logistics (Shengyang, China). He was awarded the "Excellent Youth Science Foundation Award" by the National Natural Science Foundation of China in 2003, he was named the Changjiang Scholar by China Education Ministry in 2005, and he received the National Science and Technology Invention Award (Second Grade) from the Chinese Government in 2007, among several other awards.

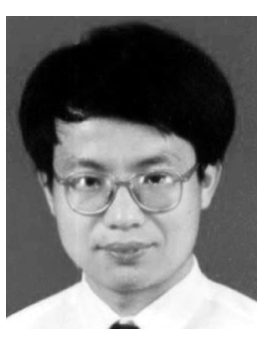

control.
Zhanshan Wang was born in Liaoning, China, in 1971. He received the M.S. degree in control theory and control engineering from Fushun Petroleum Institute, Fushun, China, in 2001 and the Ph.D. degree in control theory and control engineering from Northeastern University, Shenyang, China, in 2006.

Currently, he is an Associate Professor in the School of Information Science and Engineering, Northeastern University. His research interests include stability analysis of recurrent neural networks, fault diagnosis, fault tolerant control, and nonlinear

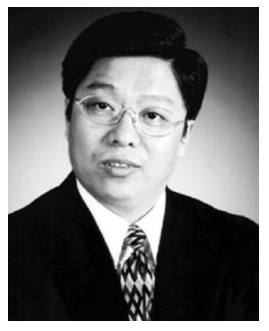

Derong Liu (S'91-M'94-SM'96-F'05) received the Ph.D. degree in electrical engineering from the University of Notre Dame, Notre Dame, IN, in 1994.

From 1993 to 1995, he was a Staff Fellow with General Motors Research and Development Center, Warren, MI. From 1995 to 1999, he was an Assistant Professor in the Department of Electrical and Computer Engineering, Stevens Institute of Technology, Hoboken, NJ. In 1999, he joined the University of Illinois at Chicago, where he became a Full Professor of Electrical and Computer Engineering and of Com- 
puter Science in 2006. He is coauthor (with A. N. Michel) of Dynamical Systems with Saturation Nonlinearities: Analysis and Design (New York: SpringerVerlag, 1994), (with A. N. Michel) Qualitative Analysis and Synthesis of Recurrent Neural Networks (New York: Marcel Dekker, 2002), (with H. G. Zhang) Fuzzy Modeling and Fuzzy Control (Cambridge, MA: Birkhauser, 2006), and (with A. N. Michel and L. Hou) Stability of Dynamical Systems: Continuous, Discontinuous, and Discrete Systems (Cambridge, MA: Birkhauser, 2008). He is coeditor (with P. J. Antsaklis) of Stability and Control of Dynamical Systems with Applications (Cambridge, MA: Birkhauser, 2003), (with F. Y. Wang) Advances in Computational Intelligence: Theory and Applications (Singapore: World Scientific, 2006), (with S. M. Fei, Z. G. Hou, H. G. Zhang, and C. Y. Sun) Advances in Neural Networks-ISNN2007 (New York: Springer-Verlag, 2007), and (with F. Y. Wang) Networked Control Systems: Theory and Applications (New York: Springer-Verlag, 2008).

Dr. Liu is an Associate Editor of Automatica. He was General Chair for the 2007 International Symposium on Neural Networks (Nanjing, China). He was a member of the Conference Editorial Board of the IEEE Control Systems Society (1995-2000), an Associate Editor of the IEEE TRANSACTIONS ON CIRCUITS AND SYSTEMS-I: FunDAMENTAL THEORY AND APPLICATIONS (1997-1999) and the IEEE TRANSACTIONS ON SigNAL PROCESSING (2001-2003), and the Letters Editor of the IEEE TRANSACTIONS ON NEURAL NETWORKS (2004-2008). Since 2004, he has been the Editor of the IEEE Computational Intelligence Society's ELECTRONIC LETTER. Currently, he is an Associate Editor of the
IEEE Transactions on Neural Networks, the IEEE Computational INTELLIGENCE MAGAZINE, and the IEEE CIRCUITS AND SYSTEMS MAGAZINE. $\mathrm{He}$ is General Chair for the 2008 IEEE International Conference on Networking, Sensing and Control (Sanya, China). He is Program Chair for the 2009 IEEE International Symposium on Adaptive Dynamic Programming and Reinforcement Learning, the 2008 International Joint Conference on Neural Networks, the 2007 IEEE International Symposium on Approximate Dynamic Programming and Reinforcement Learning, the 21st IEEE International Symposium on Intelligent Control (2006), and the 2006 International Conference on Networking, Sensing and Control. He is an elected AdCom member of the IEEE Computational Intelligence Society (2006-2008), Chair of the Chicago Chapter of the IEEE Computational Intelligence Society, Founding Chair of the Technical Committee on Adaptive Dynamic Programming and Reinforcement Learning of the IEEE Computational Intelligence Society, and Past Chair of the Technical Committee on Neural Systems and Applications of the IEEE Circuits and Systems Society. He received the Michael J. Birck Fellowship from the University of Notre Dame (1990), the Harvey N. Davis Distinguished Teaching Award from Stevens Institute of Technology (1997), the Faculty Early Career Development (CAREER) Award from the National Science Foundation (1999), and the University Scholar Award from University of Illinois (2006-2009). He is a member of Eta Kappa Nu. In 2008, he was selected into the "100 Talents Project" by the Chinese Academy of Sciences. 